\title{
Multivariable control structure design based on mixed-integer quadratic programming
}

\author{
Lautaro Braccia, ${ }^{\dagger}$ Pablo A. Marchetti, ${ }^{\ddagger}$ Patricio Luppi, ${ }^{\dagger} \uparrow$ and David \\ Zumoffen ${ }^{* \dagger, \uparrow}$ \\ $\dagger$ †rupo de Ingeniería de Sistemas de Procesos (GISP), Centro Franco-Argentino de \\ Ciencias de la Información y de Sistemas (CIFASIS), CONICET-UNR, 27 de Febrero 210 \\ bis, (S2000EZP) Rosario, Argentina. \\ $\ddagger$ Instituto de Desarrollo Tecnológico para la Industria Química (INTEC), UNL-CONICET, \\ Güemes 3450, (3000) Santa Fe, Argentina. \\ IUniversidad Nacional de Rosario, Rosario, Argentina \\ $\S$ Universidad Tecnológica Nacional - FRRo, Rosario, Argentina \\ E-mail: zumoffen@cifasis-conicet.gov.ar \\ Phone: +54-341-4237248 int. 332. Fax: +54-341-482-1772
}

\begin{abstract}
In this work a new approach to address multivariable control structure (MCS) design for medium/large-scale processes is proposed. The classical MCS design methodologies rely on superstructure representations which define sequential and/or bilevel mixed-integer nonlinear programming (MINLP) problems. The main drawbacks of this kind of approach are the complexity of the required solution methods (stochastic/deterministic global search), the computational time, and the optimality of the solution when simplifications are made. Instead, this work shows that, by using the sum of squared deviations (SSD) as well as the net load evaluation (NLE) concepts,
\end{abstract}


the control structure design problem can be formulated as a mixed-integer quadratic programming (MIQP) model with linear constraints, featuring both optimality and improved computational performance due to state-of-the-art solvers. The formulation is implemented in the GAMS environment using CPLEX as the selected solver and two typical case studies are presented to show the benefits of the proposed approach.

\section{Introduction}

In general, all industrial processes need to be controlled in some extent if some specific behavior is required for the plant as a whole. This specific behavior is usually related to security, economic, performance, and environmental objectives. Several decisions are required before a feasible control structure is selected. The multivariable control structure (MCS), or plantwide control (PWC), design is a critical research area which intends to address all

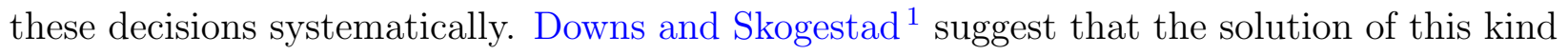
of problems requires the integration of several knowledge bases with different insights, which shows the complexity of a unified (all in one) methodology. Moreover, Stephanopoulos and Reklaitis $^{2}$ identify these complex and integrated problems as the new trends that must be addressed by the process systems engineering (PSE) community.

Any MCS design methodology should answer the following basic questions: (1) How many and which output variables should be controlled? (2) How many and which input variables need to be manipulated? (3) What is the best input-output pairing between these sets? (4) Which controller interaction (diagonal, sparse, full), policy (decentralized or centralized), technology (classical or advanced), and tuning should be selected? Most of the approaches for MCS design proposed in the literature address this problem only partially due to its inherent complexity. On the one hand, the heuristic methods become intractable for medium-large scale processes, requiring some kind of simplification (ad-hoc decisions) to reduce the overall problem size. This drawback produces, in general, suboptimal designs from the operating as well as investment cost perspectives. On the other hand, the methodologies supported 
by mathematical and/or systems theory are more suitable to deal with medium-large scale processes systematically, as well as to be integrated with other problems such as process synthesis/design. On this broad line, several contributions with the most varied themes and results appear in the literature, which include: stability/controllability/robustness from the steady-state as well as dynamic point of view ${ }^{3-5}$, input-output pairing problems for decentralized square MCS designs ${ }^{6-9}$, self-optimizing control methods focused on using all the available manipulated variables $(\mathrm{MVs})^{1,10}$, deviation-based indexes for arbitrary MCS (decentralized, sparse, full $)^{11,12}$, combinations of the former into multi-objective criteria $^{1,11,13}$, integration between heuristics and steady-state/dynamic simulations ${ }^{14,15}$, and economic plantwide control where the MCS design is integrated to optimal/safe process operation ${ }^{16-19}$. Recently, Gutierrez et al. ${ }^{20}$ proposed a control structure selection framework for simultaneous process and control design based on model predictive control (MPC), which extends a previous work of the same authors ${ }^{21}$. They introduce a mixed-integer nonlinear programming formulation, which is redefined as a nonlinear programming model by removing the discrete decision variables from the inner problem. The proposed framework handles decentralized, partially decentralized, and full control structures and, at each optimization step, requires dynamic simulations to evaluate the closed-loop performance. Most of these mathematical approaches are based on process models (dynamic/steady-state, linear/nonlinear) and mixed-integer nonlinear programming formulations, use decentralized control structures with a fixed order, and are applied to small/medium scale processes.

A unified methodology (considering all the basic questions posed above) requires some kind of superstructure representation defined by bilevel (embedded) and/or sequential mixedinteger nonlinear programming (MINLP) models. At this point two additional drawbacks appear: 1- a MINLP formulation requires complex global optimization algorithms (stochastic/deterministic) which become intractable for medium/large-scale processes ${ }^{8}$ and 2- there is a lack of rigorous global optimization algorithms for solving bilevel MINLP (BMINLP) models $^{22}$. It is clear that a unified superstructure for MCS design is essential to solve the 
overall control problem effectively and accurately. Moreover, it is important to carefully analyze the final formulation of this kind of problems because sometimes a small change in the modeling approach leads to formulations that are easier to implement and to solve. Hence, in this context, the main contributions of the present work are summarized in the next section.

\subsection{Contribution of this work}

In this work the BMINLP superstructure for MCS design presented in Zumoffen ${ }^{12}$ is successfully reformulated as a mixed-integer quadratic programming (MIQP) model. The new representation allows to solve a very complex problem with a more suitable method, i.e., by using state-of-the-art solvers such as CPLEX in GAMS. Indeed, while the new MIQP representation still requires binary/integer decision variables, it does not introduce nonconvex constraints. In this way, the use of complex MINLP global optimization algorithms (stochastic/deterministic), which present serious drawbacks related to computing time, computational resources, and optimality of the solution when the process size increases (becoming intractable for medium/large-scale plants) is avoided. Furthermore, there is a lack of rigorous global optimization algorithms for solving BMINLP models ${ }^{22}$, which supports the convenience of an alternative approach.

To the best of our knowledge, the proposed MCS design mathematical formulation is the first equation-oriented superstructure addressing simultaneously: the selection of controlled $(\mathrm{CVs})$ and manipulated variables $(\mathrm{MVs})$, the input-output pairing definition, the controller design, the initial control requisites/heuristics, and the test of stability using steady-state information, that is applicable to medium/large-scale processes.

The main contributions of this work are summarized in the following items:

- An alternative and effective MIQP formulation for MCS design applicable to medium/largescale processes is presented. 
- The BMINLP representation is avoided via a linear/convex equation-oriented superstructure.

- The MIQP formulation guarantees the global optimum solution while improving the computing time.

- Suitable implementation and solution tools are readily available (GAMS/CPLEX).

- The proposed superstructure addresses the CVs and MVs selection, the input-output pairing definition, the controller design, the heuristic concepts, and a simplified stability testing, simultaneously.

- The proposed formulation sets the foundations for future contributions integrating process control and process synthesis and design.

This work is organized as follows: Since the proposed MIQP reformulation is obtained from the general BMINLP structure, the inherent drawbacks associated to bilevel programming are reviewed in Section 2. Section 3 presents, in the context of BMINLP, an overview of the general formulation for MCS design proposed by Zumoffen ${ }^{12}$. Besides, the main concepts behind the sum of squared deviations (SSD) as well as the net load evaluation (NLE) methodologies are discussed in Sections 3.1 and 3.2, respectively. The complete MIQP reformulation is presented in Section 4, where each constitutive part, i.e., the SSD problem, the NLE approach, the RGA-based input-output problem, the stability/robustness test, and the heuristics consideration, is described in Sections 4.1, 4.2, 4.2.1, 4.2.2, and 4.3, respectively. The application of the proposed methodology to well-known medium/large-scale benchmark examples, such as the Tennessee Eastman process and the waste water treatment plant, is presented in Section 5. Finally, conclusions and the future work are discussed in Section 6. 


\section{The reformulation of bilevel programming}

Bilevel programs are defined as optimization models where two layers can be clearly identified: 1- the upper or leader program with its own associated cost function, decision variables, and constraints, and 2- the lower or follower problem which represents another complete optimization problem embedded in the constraints of the upper level. These levels can be cooperative or not depending on the nature of the problem being tackled ${ }^{22,23}$. Several challenging problems in process systems engineering (PSE) can be formulated as bilevel programming models, two typical examples being control structure selection and optimal process design and operation.

Although the literature is extensive for bilevel linear/nonlinear/quadratic programming (BLP/BNLP/BQP) approaches, which exclude the lower level from discrete decisions, there is a lack of rigorous global optimization algorithms for solving bilevel mixed-integer nonlinear programing (BMINLP) problems ${ }^{22}$. The BLP/BNLP/BQP problems can be reformulated into single-level mathematical programs by replacing the lower-level convex program with the corresponding Karush-Kuhn-Tucker (KKT) conditions. This philosophy is not applicable to BMINLP due to the discrete variables in the lower level, and some reformulations need to be used to solve this kind of problems. 
A general representation of the BMINLP problem is show in eq. (1),

$$
\begin{aligned}
& \min _{\mathbf{x}_{u}, \mathbf{z}_{u}} F\left(\mathbf{x}_{u}, \mathbf{z}_{u}, \mathbf{x}_{l}, \mathbf{z}_{l}\right) \\
& \text { s.t. } G_{i}\left(\mathbf{x}_{u}, \mathbf{z}_{u}, \mathbf{x}_{l}, \mathbf{z}_{l}\right) \leq 0, \quad i=1, \ldots, m \\
& H_{j}\left(\mathbf{x}_{u}, \mathbf{z}_{u}, \mathbf{x}_{l}, \mathbf{z}_{l}\right)=0, \quad j=1, \ldots, r \\
& {\left[\begin{array}{ll}
\min _{\mathbf{x}_{l}, \mathbf{z}_{l}} & f\left(\mathbf{x}_{u}, \mathbf{z}_{u}, \mathbf{x}_{l}, \mathbf{z}_{l}\right) \\
\text { s.t. } & g_{i^{\prime}}\left(\mathbf{x}_{u}, \mathbf{z}_{u}, \mathbf{x}_{l}, \mathbf{z}_{l}\right) \leq 0, \quad i^{\prime}=1, \ldots, m^{\prime} \\
& h_{j^{\prime}}\left(\mathbf{x}_{u}, \mathbf{z}_{u}, \mathbf{x}_{l}, \mathbf{z}_{l}\right)=0, \quad j^{\prime}=1, \ldots, r^{\prime}
\end{array}\right.} \\
& \mathbf{x}_{u} \in \mathbb{R}^{q}, \mathbf{x}_{l} \in \mathbb{R}^{q^{\prime}}, \mathbf{z}_{u} \in \mathbb{Z}^{p}, \mathbf{z}_{l} \in \mathbb{Z}^{p^{\prime}}
\end{aligned}
$$

where $F / f, G_{i} / g_{i^{\prime}}, H_{j} / h_{j^{\prime}}$ are the cost function, the inequality constraint functions, and the equality constraint functions of upper/lower levels, respectively. Furthermore, $\mathbf{x}_{u}$ and $\mathbf{z}_{u}$ are the continuous and discrete variables associated to the upper level and $\mathbf{x}_{l}$ and $\mathbf{z}_{l}$ the corresponding ones tied to the lower level.

For some small-scale cases with special structure, the problem in eq. (1) can be addressed with global optimization techniques as proposed by Yue and You ${ }^{22}$. Indeed, they present a reformulation and decomposition algorithm based on optimal value formulation and partial enumeration. Although their methodology works fine for small cases, its application to largescale problems is limited by the increasing amount of computational resources needed. The authors also analyze an alternative single-level parametric centralized formulation, which is shown in eq. (2), and illustrate the difference between the latter and the problem in eq. (1). In eq. (2), the parameter $\varepsilon$ allows to balance the emphasis between the upper and lower level cost functions. 


$$
\begin{array}{cl}
\min _{\mathbf{x}_{u}, \mathbf{z}_{u}, \mathbf{x}_{l}, \mathbf{z}_{l}} & F\left(\mathbf{x}_{u}, \mathbf{z}_{u}, \mathbf{x}_{l}, \mathbf{z}_{l}\right)+\varepsilon f\left(\mathbf{x}_{u}, \mathbf{z}_{u}, \mathbf{x}_{l}, \mathbf{z}_{l}\right) \\
\text { s.t. } & G_{i}\left(\mathbf{x}_{u}, \mathbf{z}_{u}, \mathbf{x}_{l}, \mathbf{z}_{l}\right) \leq 0, \quad i=1, \ldots, m \\
& H_{j}\left(\mathbf{x}_{u}, \mathbf{z}_{u}, \mathbf{x}_{l}, \mathbf{z}_{l}\right)=0, \quad j=1, \ldots, r \\
& g_{i^{\prime}}\left(\mathbf{x}_{u}, \mathbf{z}_{u}, \mathbf{x}_{l}, \mathbf{z}_{l}\right) \leq 0, \quad i^{\prime}=1, \ldots, m^{\prime} \\
& h_{j^{\prime}}\left(\mathbf{x}_{u}, \mathbf{z}_{u}, \mathbf{x}_{l}, \mathbf{z}_{l}\right)=0, \quad j^{\prime}=1, \ldots, r^{\prime} \\
& \mathbf{x}_{u} \in \mathbb{R}^{q}, \mathbf{x}_{l} \in \mathbb{R}^{q^{\prime}}, \mathbf{z}_{u} \in \mathbb{Z}^{p}, \mathbf{z}_{l} \in \mathbb{Z}^{p^{\prime}}
\end{array}
$$

While the formulations in eq. (1) and eq. (2) are not equivalent, the approach used in eq. (2) is very useful for some large-scale cases, since it avoids the implementation of nonlinear global optimization algorithms which are very expensive from the computational point of view. In fact, we are particularly interested in this kind of formulation due to some specific characteristics of the multivariable control structure design problem. In the following sections a complete reformulation of the BMINLP approach for MCS design is proposed.

\section{MCS design: a general formulation based on bilevel MINLP}

We consider as a starting point the bilevel mixed-integer nonlinear formulation based on combined indexes proposed by Zumoffen ${ }^{12}$. This methodology represents a complex optimization problem where the classical decisions related to the controlled and/or manipulated variables selection, the input-output pairing, robustness/stability, and the controller structure (decentralized, sparse, full) are parameterized into a superstructure via specific binary selection variables.

This approach is applicable to stable (or stabilized) processes, considering a transfer 
functions matrix (TFM) representation (Laplace domain) of the plant given by $\mathbf{y}(s)=$ $\mathbf{G}(s) \mathbf{u}(s)+\mathbf{D}(s) \mathbf{d}^{*}(s)$, which can be partitioned as:

$$
\left[\begin{array}{l}
\mathbf{y}_{\mathrm{s}}(s) \\
\mathbf{y}_{\mathrm{r}}(s)
\end{array}\right]=\left[\begin{array}{ll}
\mathbf{G}_{\mathrm{s}}(s) & \mathbf{G}_{\mathrm{s}}^{*}(s) \\
\mathbf{G}_{\mathrm{r}}(s) & \mathbf{G}_{\mathrm{r}}^{*}(s)
\end{array}\right]\left[\begin{array}{c}
\mathbf{u}_{\mathrm{s}}(s) \\
\mathbf{u}_{\mathrm{r}}(s)
\end{array}\right]+\left[\begin{array}{c}
\mathbf{D}_{\mathrm{s}}(s) \\
\mathbf{D}_{\mathrm{r}}(s)
\end{array}\right] \mathbf{d}^{*}(s)
$$

where $\mathbf{y}(s)$ are the potential output measurements, $\mathbf{u}(s)$ are the available MVs, and $\mathbf{d}^{*}(s)$ are the disturbance variables $(\mathrm{DVs})$ with size $(m \times 1),(n \times 1)$, and $(p \times 1)$, respectively. For some particular partitioning of $\mathbf{G}(s)$, as shown in eq. (3), $\mathbf{y}_{\mathbf{s}}(s)$ and $\mathbf{y}_{\mathrm{r}}(s)$ are the selected controlled variables $(\mathrm{CVs})$ and uncontrolled variables $(\mathrm{UVs})$, respectively. Besides, $\mathbf{u}_{\mathrm{s}}(s)$ are the selected manipulated variables (MVs) to control the subprocess $\mathbf{G}_{\mathrm{s}}(s)$ and $\mathbf{u}_{\mathrm{r}}(s)=0$ are not used. It is worth noting that a normalized version of the process model is used in this work. Although there are several ways to perform the scaling of the process, generally accepted procedures are to develop linear models based on two different approaches: 1working with deviations/incremental variables and scaling against the maximum expected value and 2- when data from some identification experiment are available, to normalize all the variables to zero mean and unit variance. The latter normalizing approach is adopted in this work. Hence, the potential changes in set points and disturbances are considered to be unitary because all the variables are assumed to be equally important. While the SSD and NLE indexes presented in the following sections are scaling dependent, this dependency is not a negative property. It only means that the selected scaling should be taken into account when the results are analyzed. Previous works of the authors ${ }^{11,12,24}$ address the potential modifications of the process scaling to consider unnormalized plants and/or to define the relative degree of importance among the process variables. Considering the process partitioning given in eq. (3), the methodology of Zumoffen ${ }^{12}$ is summarized by eq. (4) and its main concepts and drawbacks are highlighted in the following paragraphs. 


$$
\begin{aligned}
& \min _{\mathbf{z}^{\mathrm{I}}, \mathbf{z}^{\mathrm{O}}, \mathbf{z}^{\mathrm{S}}}[\mathrm{SSD}+\mathrm{NLE}] \\
& \text { s.t. } \quad \mathrm{SSD}=\left\|\mathbf{G}_{\mathrm{r}} \mathbf{G}_{\mathrm{s}}^{-1}\right\|_{F}^{2}+\left\|\left(\mathbf{D}_{\mathrm{r}}-\mathbf{G}_{\mathrm{r}} \mathbf{G}_{\mathrm{s}}^{-1} \mathbf{D}_{\mathrm{s}}\right)\right\|_{F}^{2} \\
& \mathrm{NLE}=\left\|\mathbf{I}-\tilde{\mathbf{G}}_{\mathrm{s}} \mathbf{G}_{\mathrm{s}}^{-1}\right\|_{F}^{2}+\left\|\tilde{\mathbf{G}}_{\mathrm{s}} \mathbf{G}_{\mathrm{s}}^{-1} \mathbf{D}_{\mathrm{s}}\right\|_{F}^{2} \\
& q \leq \min \{m, n\} \\
& \left\|\mathbf{z}^{\mathrm{I}}\right\|_{1}=\left\|\mathbf{z}^{\mathrm{O}}\right\|_{1}=q \\
& \mathbf{T}_{\mathrm{O}}=\operatorname{nre}\left[\operatorname{diag}\left(\mathbf{z}^{\mathrm{O}}\right)\right], \mathbf{T}_{\mathrm{I}}=\operatorname{nce}\left[\operatorname{diag}\left(\mathbf{z}^{\mathrm{I}}\right)\right] \\
& \mathrm{G}_{\mathrm{s}}=\mathrm{T}_{\mathrm{O}} \mathrm{GT}_{\mathrm{I}}, \mathrm{D}_{\mathrm{s}}=\mathbf{T}_{\mathrm{O}} \mathbf{D} \\
& \mathbf{G}_{\mathrm{r}}=\overline{\mathbf{T}}_{\mathrm{O}} \mathrm{GT}_{\mathrm{I}}, \mathrm{D}_{\mathrm{r}}=\overline{\mathbf{T}}_{\mathrm{O}} \mathbf{D} \\
& \tilde{\mathbf{G}}_{\mathrm{S}}=\mathbf{G}_{\mathrm{s}} \otimes\left(\mathbf{z}^{\mathrm{P}}+\mathbf{z}^{\mathrm{S}}\right) \\
& \operatorname{Re}\left[\lambda_{i}\left(\mathbf{G}_{\mathbf{s}}\left(\tilde{\mathbf{G}}_{\mathrm{s}}\right)^{-1}\right)\right]>0, i=1, \ldots, q \\
& \sum_{i} \sum_{j} \mathbf{z}^{\mathrm{S}}(i, j) \mathbf{z}^{\mathrm{P}}(i, j)=0 \\
& \mathbf{z}^{\mathrm{I}} \in \mathbb{B}^{m}, \mathbf{z}^{\mathrm{O}} \in \mathbb{B}^{n}, \mathbf{z}^{\mathrm{S}} \in \mathbb{B}^{q \times q} \\
& {\left[\begin{array}{ll}
\min _{\mathbf{z}^{\mathrm{P}}} & \Lambda_{n} \\
\text { s.t. } & \Lambda=\mathbf{G}_{\mathrm{s}} \otimes\left(\mathbf{G}_{\mathrm{s}}^{-1}\right)^{T} \\
& \Lambda_{\mathrm{n}}=\left\|\mathbf{z}^{\mathrm{P}}-\Lambda\right\|_{\text {sum }} \\
& \sum_{i} \mathbf{z}^{\mathrm{P}}(i, j)=\sum_{j} \mathbf{z}^{\mathrm{P}}(i, j)=1 \\
& \mathbf{z}^{\mathrm{P}} \in \mathbb{B}^{q \times q}
\end{array}\right.}
\end{aligned}
$$

In Zumoffen ${ }^{12}$ the BMINLP model shown in eq. (4) has been solved using a stochastic global search method, i.e., genetic algorithms, in the Matlab context. Due to its intrinsic combinatorial complexity, this methodology has only been tested on the design of decentralized MCS. The approach is based on the combination of two steady-state indexes: the SSD, 
associated to the selection of controlled and manipulated variables, and the NLE, associated to the definition of the controller structure. It is worth mentioning that these indexes are scaling dependent, so if the normalization procedure is changed the optimal solution can also differ. To contextualize the general approach, brief comments are given about these indexes in the following sections.

\subsection{Selection of CVs and MVs}

Let us assume that the subprocess $\mathbf{G}_{\mathbf{s}}(s)$ in Eq. (3) is controlled with some structure based on integral action (1-DOF), then at steady-state $(s=0)$ we have $\mathbf{y}_{\mathrm{s}}=\mathbf{y}_{\mathrm{s}}^{\mathrm{sp}}$ and,

$$
\begin{aligned}
& \mathbf{u}_{\mathrm{s}}=\mathrm{G}_{\mathrm{s}}^{-1} \mathbf{y}_{\mathrm{s}}^{\mathrm{sp}}-\mathrm{G}_{\mathrm{s}}^{-1} \mathbf{D}_{\mathrm{s}} \mathbf{d}^{*}=\mathbf{u}_{\mathrm{s}}^{\mathrm{sp}}+\mathbf{u}_{\mathrm{s}}^{\mathrm{d}}, \\
& \mathbf{y}_{\mathrm{r}}=\mathbf{G}_{\mathrm{r}} \mathbf{G}_{\mathrm{s}}^{-1} \mathbf{y}_{\mathrm{s}}^{\mathrm{sp}}+\left(\mathbf{D}_{\mathrm{r}}-\mathbf{G}_{\mathrm{r}} \mathbf{G}_{\mathrm{s}}^{-1} \mathbf{D}_{\mathrm{s}}\right) \mathbf{d}^{*}=\mathbf{y}_{\mathrm{r}}^{\mathrm{sp}}+\mathbf{y}_{\mathrm{r}}^{\mathrm{d}}
\end{aligned}
$$

where $\mathbf{u}_{\mathrm{s}}^{\mathrm{sp}}=\mathbf{G}_{\mathrm{s}}^{-1} \mathbf{y}_{\mathrm{s}}^{\mathrm{sp}}$ is the MVs component associated to changes of set-point and $\mathbf{u}_{\mathrm{s}}^{\mathrm{d}}=$ $-\mathbf{G}_{\mathrm{s}}^{-1} \mathbf{D}_{\mathrm{s}} \mathbf{d}^{*}$ the MVs component tied to the disturbance effects. Similarly, the uncontrolled variables can be partitioned as $\mathbf{y}_{\mathrm{r}}^{\mathrm{sp}}=\mathbf{G}_{\mathrm{r}} \mathbf{u}_{\mathrm{s}}^{\mathrm{sp}}$ and $\mathbf{y}_{\mathrm{r}}^{\mathrm{d}}=\mathbf{G}_{\mathrm{r}} \mathbf{u}_{\mathrm{s}}^{\mathrm{d}}+\mathbf{D}_{\mathrm{r}} \mathbf{d}^{*}$. Thus, the SSD index in eq. (4b) quantifies the deviations of the uncontrolled variables $\mathbf{y}_{\mathbf{r}}$ from their nominal operating points when set points and disturbances occur in the plant individually. The SSD minimization properties were extensively analyzed in previous works of the authors ${ }^{12,24}$ and it was shown there that this minimization tends to maximize the minimum singular value of the subprocess $\mathbf{G}_{s}$, i.e., a well-conditioned subprocess that is easy to control is obtained ${ }^{3,4}$.

Eqs. (4d)-(4h) allow to select specific parts of the original plant model $\mathbf{G}$ and $\mathbf{D}$ in order to perform the SSD index evaluation. The transformation matrices $\mathbf{T}_{\mathrm{O}}$ and $\mathbf{T}_{\mathrm{I}}$ depend on the binary decision variables $\mathbf{z}^{\mathrm{O}}$ and $\mathbf{z}^{\mathrm{I}}$, respectively. The entries in the vector $\mathbf{z}^{\mathrm{O}}$ with unitary value indicate the output variables selected to be controlled (CVs). Similarly, the vector $\mathbf{z}^{\mathrm{I}}$ indicates the selection of the MVs. The number of unitary entries in both sets need to be the same $(q)$. Functions nre $(\cdot)$ and nce $(\cdot)$ take a square matrix and eliminate the zero rows 
and columns, respectively.

\subsection{Controller structure}

Once the model partition in eq. (3) is defined, a multivarible controller for $\mathbf{G}_{\mathbf{s}}(s)$ needs to be designed. Let us assume, without lost of generality, that internal model control (IMC) is used to this end. Thus, the controller is given by $\mathbf{G}_{\mathrm{c}}(s)=\tilde{\mathbf{G}}_{\mathrm{s}}^{-1}(s) \mathbf{F}(s)$, where $\tilde{\mathbf{G}}_{\mathbf{s}}(s)$ is a particular selection of the entries of $\mathbf{G}_{\mathbf{s}}(s)$ and $\mathbf{F}(s)$ a low-pass diagonal filter matrix. It is assumed here that the selection of $\tilde{\mathbf{G}}_{\mathrm{s}}(s)$ is performed on the invertible part of $\mathbf{G}_{\mathrm{s}}(s)$ according to the procedure opportunely defined by Garcia and Morari ${ }^{25}$. Moreover, in a previous work of the authors ${ }^{24}$ this approach is discussed and some conservative tuning rules are suggested for the diagonal filter matrix $\mathbf{F}(s)$. In this context, the closed-loop relationship on the $\mathrm{CVs}$ is:

$$
\mathbf{y}_{\mathrm{s}}(s)=\mathbf{F}(s) \mathbf{y}_{\mathrm{s}}^{\mathrm{sp}}(s)+(\mathbf{I}-\mathbf{F}(s)) \mathbf{y}_{\mathrm{s}}^{\text {net }}(s)
$$

where

$$
\begin{aligned}
\mathbf{y}_{\mathrm{s}}^{\text {net }}(s) & =\mathbf{A}_{\mathrm{n}}(s) \mathbf{y}_{\mathrm{s}}(s)+\mathbf{B}_{\mathrm{n}}(s) \mathbf{d}^{*}(s) \\
\mathbf{A}_{\mathrm{n}}(s) & =\left[\mathbf{I}+\left(\mathbf{G}_{\mathrm{s}}(s)-\tilde{\mathbf{G}}_{\mathrm{s}}(s)\right) \mathbf{G}_{\mathrm{c}}(s)\right]^{-1}\left(\mathbf{G}_{\mathrm{s}}(s)-\tilde{\mathbf{G}}_{\mathrm{s}}(s)\right) \mathbf{G}_{\mathrm{c}}(s) \\
\mathbf{B}_{\mathrm{n}}(s) & =\left[\mathbf{I}+\left(\mathbf{G}_{\mathrm{s}}(s)-\tilde{\mathbf{G}}(s)\right) \mathbf{G}_{\mathrm{c}}(s)\right]^{-1} \mathbf{D}_{\mathbf{s}}(s),
\end{aligned}
$$

being $\mathbf{y}_{\mathrm{s}}^{\text {net }}(s)$ the so-called net load effect, which quantifies the multivariable interaction at closed-loop. It is clear from eq. (6) that the term associated to the net load effect is an undesired disturbance from the CVs point of view. An extensive analysis of this term can be found in previous works ${ }^{12,24}$ where a new index called net load evaluation (NLE) is proposed by minimizing $\mathbf{y}_{\mathrm{s}}^{\text {net }}$ at steady-state

$$
\mathbf{y}_{\mathrm{s}}^{\mathrm{net}}=\left(\mathbf{I}-\tilde{\mathbf{G}}_{\mathrm{s}} \mathbf{G}_{\mathrm{s}}^{-1}\right) \mathbf{y}_{\mathrm{s}}^{\mathrm{sp}}+\left(\tilde{\mathbf{G}}_{\mathrm{s}} \mathbf{G}_{\mathrm{s}}^{-1} \mathbf{D}_{\mathrm{s}}\right) \mathbf{d}^{*}=\mathbf{y}_{\mathrm{s}}^{\mathrm{sp}}-\tilde{\mathbf{G}}_{\mathrm{s}} \mathbf{u}_{\mathrm{s}}^{\mathrm{sp}}-\tilde{\mathbf{G}}_{\mathrm{s}} \mathbf{u}_{\mathrm{s}}^{\mathrm{d}}
$$


using a sum of squared deviations as shown in eq. (4c). Basically, the NLE approach allows to define a specific plant-model mismatch to develop the best IMC controller interaction improving the closed-loop performance.

The mentioned plant-model mismatch selection is performed in eq. (4i) via $\mathbf{z}^{\mathrm{P}}$ and $\mathbf{z}^{\mathrm{S}}$, which represent binary decision matrices of $q \times q$. Matrix $\mathbf{z}^{\mathrm{P}}$ represents the best decentralized input-output pairing from the embedded minimization in eq. (4m) and $\mathbf{z}^{\mathrm{S}}$ is the sparse model selection given around $\mathbf{z}^{\mathrm{P}}$ to minimize the NLE index. The $\mathbf{z}^{\mathrm{P}}$ selection in eq. $(4 \mathrm{~m})$ is based on the so-called RGA-number $\Lambda_{\mathrm{n}}=\left\|\mathbf{z}^{\mathrm{P}}-\Lambda\right\|_{\text {sum }}$ which in turn depends on the classical relative gain array (RGA), $\Lambda=\mathbf{G}_{\mathrm{s}} \otimes\left(\mathbf{G}_{\mathrm{s}}^{-1}\right)^{T}$. Equation (4k) represents a structural constraint and eq. (4j) is the so-called stability/robustness criterion developed by Garcia and Morari ${ }^{25}$ for multivariable control structures based on IMC theory, where Re[·] is the real part function and $\lambda_{i}(\cdot)$ is the $i$-th eigenvalue.

Note 1: It is worth mentioning that an alternative MCS design procedure to eq. (4) was proposed in Zumoffen (2013) ${ }^{11}$ using a suboptimal algorithmic solution method based on GA in Matlab. In Zumoffen (2016) ${ }^{12}$ the BMINLP shown in eq. (4) is solved only for decentralized control structures, also using a global GA solver. Although the approach proposed in Zumoffen (2016) ${ }^{12}$ improves the optimality degree of the solutions respect to those given in Zumoffen (2013) ${ }^{11}$, the computing time of the former is very restrictive when full or sparse control structures are needed. This behavior is a typical drawback of GA solutions, making these stochastic global search algorithms unpractical or even unviable for medium/large-scale processes.

\subsection{Reformulation based on single level MINLP}

Following the approach outlined in section 2, the first step to reformulate the BMINLP in eq. (4) into a single MINLP is by eliminating the embedded minimization in eq. (4m). The main idea here is to relax the optimality condition of the solution given by eq. $(4 \mathrm{~m})$, which is replaced by a feasibility condition transforming the problem into a single-level programming 
model. Besides, the parametric centralized approach is not used. Therefore, the bilevel MINLP in eq. (4) is modified into the single-level structure shown in eq. (11).

$$
\begin{aligned}
\min _{\mathbf{z}^{\mathrm{I}}, \mathbf{z}^{\mathrm{O}}, \mathbf{z}^{\mathrm{S}}, \mathbf{z}^{\mathrm{P}}} & {[\mathrm{SSD}+\mathrm{NLE}] } \\
\text { s.t. } & \text { eq. (4b) to eq. (4l) } \\
& \Lambda=\mathbf{G}_{\mathrm{s}} \otimes\left(\mathbf{G}_{\mathrm{s}}^{-1}\right)^{T} \\
& \Lambda_{\mathrm{p}}=\Lambda \otimes \mathbf{z}^{\mathrm{P}} \\
& \delta_{1} \leq \sum_{j} \Lambda_{\mathrm{p}}(i, j) \leq \delta_{2}, i=1, \ldots, q \\
& \sum_{i} \mathbf{z}^{\mathrm{P}}(i, j)=\sum_{j} \mathbf{z}^{\mathrm{P}}(i, j)=1 \\
& \mathbf{z}^{\mathrm{P}} \in \mathbb{B}^{q \times q}
\end{aligned}
$$

The constraints in eqs. (11d) and (11e) guarantee that the selected input-output pairing given by $\mathbf{z}^{\mathrm{P}}$ is a feasible solution, where $\Lambda_{\mathrm{p}}$ contains the entries selected by $\mathbf{z}^{\mathrm{P}}$ from the RGA and zero elsewhere. In eq. (11e), $\delta_{1}$ and $\delta_{2}$ are design parameters which allow to define the desired amount of interaction (minimum and maximum, respectively) between inputs and outputs to be paired in a decentralized control structure. Besides, the binary decision matrix $\mathbf{z}^{\mathrm{P}}$ is now an argument of the main/single minimization problem together with $\mathbf{z}^{\mathrm{I}}$, $\mathbf{z}^{\mathrm{O}}$, and $\mathbf{z}^{\mathrm{S}}$.

This reformulation is based on the following reasoning: the optimization in eq. (4m) generates the most decentralized input-output pairing for a given subprocess $\mathbf{G}_{\mathrm{s}}$, i.e. the RGA has entries very close to unity for the paired elements and features very low values for the remaining entries. From the control point of view this means good control characteristics, low control energy, and low interaction at closed loop. Analyzing the inherent properties of the SSD and NLE approaches it can be observed that, on the one hand, from eq. (5) the SSD minimization tends to maximize the minimum singular value of the process $\mathbf{G}_{\mathrm{s}}$ by minimizing 
the deviations of $\mathbf{u}_{\mathrm{s}}^{\mathrm{sp}}$ and $\mathbf{u}_{\mathrm{s}}^{\mathrm{d}}$ (i.e., a well conditioned process, good control characteristics, low control energy and reliable RGA) and, on the other hand, the NLE minimization tends to minimize the multivariable interaction at closed loop (eq. (10)) by minimizing the multivariable gain of the process model $\tilde{\mathbf{G}}_{\mathrm{s}}$ and the deviations of $\mathbf{u}_{\mathrm{s}}^{\mathrm{sp}}$ and $\mathbf{u}_{\mathrm{s}}^{\mathrm{d}} \cdot{ }^{12,24}$ In other words, the SSD+NLE combined index (by itself) drives the optimization towards a well-conditioned subprocess with reliable RGA and minimum control energy. This will become evident in the

next section when the link between the RGA and the $\mathbf{u}_{\mathrm{s}}^{\mathrm{sp}}$ deviations are explicitly defined. Hence, the optimization in eq. $(4 \mathrm{~m})$ is replaced by a feasibility condition where two new parameters are added to handle the RGA interaction level desired by the user.

It is important to note that the formulation in eq. (11) is an intermediate step between the model presented in eq. (4) and the reformulation proposed in the following section. The optimization problem in eq. (11) is not solved in this work.

\section{Complete problem reformulation based on MIQP}

The single-level MINLP model proposed in eq. (11) is substantially modified here. It is clear that the superstructure formulation proposed in Zumoffen ${ }^{12}$, which is based on selecting specific parts of $\mathbf{G}$ and $\mathbf{D}$ via transformation matrices, generates bilinear/nonlinear terms in both the cost function and the constraints due to products between the decision variables and the matrix inverse. To overcome this situation, a different modeling approach is proposed. The main idea is to translate the selection criteria based on the process matrices ( $\mathbf{G}$ and D) to the input-output process variables $(\mathbf{u}$ and $\mathbf{y}$ ). The new representation maintains the linearity and convexity of the model and, if deterministic solvers such as CPLEX in the GAMS environment are used, guarantees that the best solution found is also the global optimum.

According to the analysis presented in Sections 3.1 and 3.2, both the SSD and the NLE indexes quantify the deviations of some specific variables from the nominal operating point 
when set points and disturbances occur individually (based on the superposition principle). Obviously, parameterized with some CVs, MVs, and controller structure selection. Thus, for each set point and disturbance change the systems of equations for $\mathbf{y}_{\mathrm{r}}$ in eq. (5) and $\mathbf{y}_{\mathrm{s}}^{\text {net }}$ in eq. (10) can be solved separately and the squared deviations added up into the cost function. The proposed new mixed-integer quadratic programming (MIQP) formulation is show in eq. (12) and described step by step in the following sections. The decision variable vectors and matrices used in the model have the following characterization:

- binary vector variables: $\mathbf{z}^{\mathrm{I}} \in \mathbb{B}^{n \times 1}$, and $\mathbf{z}^{\mathrm{O}} \in \mathbb{B}^{m \times 1}$,

- binary matrix variables: $\mathbf{z}^{\mathrm{n}}, \mathbf{z}^{\text {nd }} \in \mathbb{B}^{m \times n}$,

- real (continuous) vector variables: $\mathbf{y}_{i}^{\mathrm{c}}, \mathbf{y}_{i}^{\mathrm{nc}}, \mathbf{y}_{j}^{\mathrm{d}}, \mathbf{y}_{j}^{\mathrm{nd}} \in \mathbb{R}^{m \times 1}$, and $\mathbf{u}_{i}^{\mathrm{c}}, \mathbf{u}_{j}^{\mathrm{d}} \in \mathbb{R}^{n \times 1}$, and

- real (continuous) matrix variables: $\mathbf{u}_{i}^{\mathrm{nc}}, \mathbf{u}_{j}^{\mathrm{nd}}, \mathbf{y}^{\mathrm{r}} \in \mathbb{R}^{m \times n}$,

where $\mathbf{y}_{i}^{\mathrm{c}}, \mathbf{y}_{i}^{\mathrm{nc}}, \mathbf{u}_{i}^{\mathrm{c}}$, and $\mathbf{u}_{i}^{\mathrm{nc}}$ are defined for each $i$, and $\mathbf{y}_{j}^{\mathrm{d}}, \mathbf{y}_{j}^{\text {nd }}, \mathbf{u}_{j}^{\mathrm{d}}$, and $\mathbf{u}_{j}^{\text {nd }}$ are defined for each $j$, with $i=1, \ldots, m, l=1, \ldots, n$, and $j=1, \ldots, p$. The notation $\mathbf{A}(:, i)$ means the selection of the $i$-th column of the matrix A. Besides, $\mathbf{v}_{i}$ and $\mathbf{v}_{j}$ refer to the unit vectors of directions $i$ and $j$, respectively, for the spaces $\mathbb{R}^{m}$ and $\mathbb{R}^{p}$. While eq. (12) is presented in matrix form, a detailed description using scalar components is presented in the Appendix. 


$$
\begin{aligned}
& \min _{\mathbf{u}, \mathbf{y}, \mathbf{z}} \sum_{i=1}^{m}\left\|\mathbf{y}_{i}^{\mathrm{c}}\right\|_{2}^{2}+\sum_{j=1}^{p}\left\|\mathbf{y}_{j}^{\mathrm{d}}\right\|_{2}^{2}+\sum_{i=1}^{m}\left\|\mathbf{y}_{i}^{\mathrm{nc}}\right\|_{2}^{2}+\sum_{j=1}^{p}\left\|\mathbf{y}_{j}^{\mathrm{nd}}\right\|_{2}^{2} \\
& \text { subject to } \quad-M\left(\mathbf{1}_{m}-\mathbf{z}^{\mathrm{O}}\right) \leq \mathbf{G u}_{i}^{\mathrm{c}}-\mathbf{v}_{i} \leq M\left(\mathbf{1}_{m}-\mathbf{z}^{\mathrm{O}}\right) \\
& -M\left(\mathbf{1}_{m}-\mathbf{z}^{\mathrm{O}}\right) \leq \mathbf{G} \mathbf{u}_{j}^{\mathrm{d}}+\mathbf{D}(:, j) \leq M\left(\mathbf{1}_{m}-\mathbf{z}^{\mathrm{O}}\right) \\
& -M \mathbf{z}^{\mathrm{O}}(i) \mathbf{1}_{n} \leq \mathbf{u}_{i}^{\mathrm{c}} \leq M \mathbf{z}^{\mathrm{O}}(i) \mathbf{1}_{n} \\
& -M \mathbf{z}^{\mathrm{I}} \leq \mathbf{u}_{i}^{\mathrm{c}} \leq M \mathbf{z}^{\mathrm{I}} \\
& -M \mathbf{z}^{\mathrm{I}} \leq \mathbf{u}_{j}^{\mathrm{d}} \leq M \mathbf{z}^{\mathrm{I}} \\
& \mathbf{y}_{i}^{\mathrm{c}}-\mathbf{G u}_{i}^{\mathrm{c}}+\mathbf{v}_{i} \otimes \mathbf{z}^{\mathrm{O}}=\mathbf{0}_{m} \\
& \mathbf{y}_{j}^{\mathrm{d}}-\mathbf{G u}_{j}^{\mathrm{d}}-\mathbf{D}(:, j)=\mathbf{0}_{m} \\
& \left\|\mathbf{z}^{\mathrm{O}}\right\|_{1}=\left\|\mathbf{z}^{\mathrm{I}}\right\|_{1} \\
& \left\|\mathbf{z}^{\mathrm{O}}\right\|_{1} \leq q^{\max } \\
& -M\left(\mathbf{1}_{m \times n}-\mathbf{z}^{\mathrm{n}}\right) \leq \mathbf{u}_{i}^{\mathrm{nc}}-\mathbf{1}_{m}\left(\mathbf{u}_{i}^{\mathrm{c}}\right)^{T} \leq M\left(\mathbf{1}_{m \times n}-\mathbf{z}^{\mathrm{n}}\right) \\
& -M\left(\mathbf{1}_{m \times n}-\mathbf{z}^{\mathrm{n}}\right) \leq \mathbf{u}_{j}^{\mathrm{nd}}-\mathbf{1}_{m}\left(\mathbf{u}_{j}^{\mathrm{d}}\right)^{T} \leq M\left(\mathbf{1}_{m \times n}-\mathbf{z}^{\mathrm{n}}\right) \\
& -M \mathbf{z}^{\mathrm{n}} \leq \mathbf{u}_{i}^{\mathrm{nc}} \leq M \mathbf{z}^{\mathrm{n}} \\
& -M \mathbf{z}^{\mathrm{n}} \leq \mathbf{u}_{j}^{\mathrm{nd}} \leq M \mathbf{z}^{\mathrm{n}} \\
& \mathbf{y}_{i}^{\mathrm{nc}}+\left(\mathbf{G} \otimes \mathbf{u}_{i}^{\mathrm{nc}}\right) \mathbf{1}_{n}-\mathbf{v}_{i} \otimes \mathbf{z}^{\mathrm{O}}=\mathbf{0}_{m} \\
& \mathbf{y}_{j}^{\mathrm{nd}}+\left(\mathbf{G} \otimes \mathbf{u}_{j}^{\mathrm{nd}}\right) \mathbf{1}_{n}=\mathbf{0}_{m} \\
& \mathbf{z}^{\mathrm{n}}(i, l) \leq \mathbf{z}^{\mathrm{I}}(l), \mathbf{z}^{\mathrm{n}}(i, l) \leq \mathbf{z}^{\mathrm{O}}(i) \\
& \mathbf{z}^{\mathrm{I}}(l) \leq\left\|\mathbf{z}^{\mathrm{n}}(:, l)\right\|_{1}, \mathbf{z}^{\mathrm{O}}(i) \leq\left\|\mathbf{z}^{\mathrm{n}}(i,:)^{T}\right\|_{1} \\
& \mathbf{z}^{\mathrm{I}}(l)=\left\|\mathbf{z}^{\text {nd }}(:, l)\right\|_{1}, \mathbf{z}^{\mathrm{I}}(l)=\left\|\mathbf{z}^{\text {nd }}(i,:)^{T}\right\|_{1} \\
& \mathbf{z}^{\text {nd }} \leq \mathbf{z}^{\text {n }} \\
& -M\left(\mathbf{1}_{m \times n}-\mathbf{z}^{\mathrm{nd}}\right) \leq \mathbf{y}^{\mathrm{r}}-\mathbf{G} \otimes\left[\mathbf{u}_{1}^{\mathrm{c}}, \ldots, \mathbf{u}_{m}^{\mathrm{c}}\right]^{T} \leq M\left(\mathbf{1}_{m \times n}-\mathbf{z}^{\mathrm{nd}}\right) \\
& \delta_{1} \mathbf{z}^{\text {nd }} \leq \mathbf{y}^{\mathrm{r}} \leq \delta_{2} \mathbf{z}^{\text {nd }} \\
& \sum_{i=1}^{m}\left(\mathbf{z}^{\mathrm{O}}(i)-\mathbf{y}_{i}^{\mathrm{nc}}(i)\right) \geq \delta
\end{aligned}
$$




\subsection{The SSD subproblem}

The reformulation in eq. (12) avoids the matrix partitioning defined in eqs. (4f) to (4h) as well as the complex nonlinear matrix operations in eq. (4b) by parameterizing the control superstructure directly on the complete process model $\mathbf{y}=\mathbf{G u}+\mathbf{D d}^{*}$. If set points and disturbances are considered separately, the following systems of equations for each set point change $i$ and for each disturbance change $j$ are considered:

$$
\begin{aligned}
& \text { Set point changes: } \begin{array}{l}
\mathbf{y}_{i}^{\mathrm{c}}=\mathbf{G} \mathbf{u}_{i}^{\mathrm{c}}-\mathbf{v}_{i} \\
\text { selected CVs in } \mathbf{y}_{i}^{\mathrm{c}} \text { fixed to zero } \\
\text { non selected MVs in } \mathbf{u}_{i}^{\mathrm{c}} \text { fixed to zero }
\end{array} \\
& \text { Disturbance changes: }\left[\begin{array}{l}
\mathbf{y}_{j}^{\mathrm{d}}=\mathbf{G u}_{j}^{\mathrm{d}}+\mathbf{D} \mathbf{v}_{j} \\
\text { selected CVs in } \mathbf{y}_{j}^{\mathrm{d}} \text { fixed to zero } \\
\text { non selected MVs in } \mathbf{u}_{j}^{\mathrm{d}} \text { fixed to zero }
\end{array} \quad \forall j=1, \ldots, p\right.
\end{aligned}
$$

In eq. (13), $\mathbf{u}_{i}^{\mathrm{c}}$ and $\mathbf{u}_{j}^{\mathrm{d}}$ are real (continuous) vector variables of size $n \times 1$ associated to the input response for the $i$-th set point change and the $j$-th disturbance change, respectively. Similarly, $\mathbf{y}_{i}^{\mathrm{c}}$ and $\mathbf{y}_{j}^{\mathrm{d}}$ are real (continuous) vector variables of size $m \times 1$ associated to the deviations of the uncontrolled variables (UVs). In turn, the unitary vectors $\mathbf{v}_{i}$ (of size $m \times 1$ ) and $\mathbf{v}_{j}(p \times 1)$ generate the (normalized) changes of set point $i$ and disturbance $j$. In order to use the systems in eq. (13), specific entries of the input $\left(\mathbf{u}_{i}^{\mathrm{c}}\right.$ and $\left.\mathbf{u}_{j}^{\mathrm{d}}\right)$ and output $\left(\mathbf{y}_{i}^{\mathrm{c}}\right.$ and $\mathbf{y}_{j}^{\mathrm{d}}$ ) vectors should be fixed depending on the selection of MVs and CVs. In particular, the entries associated to input variables not manipulated and output variables being controlled should be zero.

In the proposed model, the SSD subproblem is addressed by eqs. (12b) to (12j). The variables $\left(\mathbf{y}_{i}^{\mathrm{c}}, \mathbf{u}_{i}^{\mathrm{c}}\right)$ and $\left(\mathbf{y}_{j}^{\mathrm{d}}, \mathbf{u}_{j}^{\mathrm{d}}\right)$ contain the required solutions of the systems of equations for the $i$-th set point and the $j$-th disturbance change, respectively. These solutions are obtained 
according to the CVs and MVs selected by the binary decision variables $\mathbf{z}^{\mathrm{O}}$ and $\mathbf{z}^{\mathrm{I}}$.

In order to explain how the constraints associated to set point changes are modeled, i.e., eqs. (12b), (12d), (12e), and (12g), let us assume that the binary vectors $\mathbf{z}^{\mathrm{I}}$ and $\mathbf{z}^{\mathrm{O}}$ have fixed binary values with $\left\|\mathbf{z}^{\mathrm{O}}\right\|_{1}=\left\|\mathbf{z}^{\mathrm{I}}\right\|_{1}=q$ and, therefore, determine a selection of manipulated and controlled variables, respectively. If $\mathbf{T}_{\mathrm{O}}$ and $\mathbf{T}_{\mathrm{I}}$ are defined according to eq. (4f) and we define

$$
\mathbf{P}=\left[\begin{array}{c}
\mathbf{T}_{\mathrm{O}} \\
\overline{\mathbf{T}}_{\mathrm{O}}
\end{array}\right], \quad \mathbf{Q}=\left[\begin{array}{ll}
\mathbf{T}_{\mathrm{I}} & \overline{\mathbf{T}}_{\mathrm{I}}
\end{array}\right]
$$

then, by construction, the following relations hold:

$$
\mathbf{P} \mathbf{1}_{m}=\mathbf{1}_{m}, \quad \mathbf{P} \mathbf{z}^{\mathrm{O}}=\left[\begin{array}{c}
\mathbf{1}_{q} \\
\mathbf{0}_{m^{\prime}}
\end{array}\right], \quad \mathbf{Q} \mathbf{Q}^{T}=\mathbf{I}_{n}, \quad \text { and } \quad \mathbf{Q}^{T} \mathbf{z}^{\mathrm{I}}=\left[\begin{array}{c}
\mathbf{1}_{q} \\
\mathbf{0}_{n^{\prime}}
\end{array}\right]
$$

where $m^{\prime}=m-q$ and $n^{\prime}=n-q$. Using eq. (15), if eq. (12b) is pre-multiplied by $\mathbf{P}$ and the identity $\mathbf{G} \mathbf{u}_{i}^{\mathrm{c}}=\mathbf{G}\left(\mathbf{Q} \mathbf{Q}^{T}\right) \mathbf{u}_{i}^{\mathrm{c}}$ is considered the following equation is obtained:

$$
-M\left[\begin{array}{c}
\mathbf{0}_{q} \\
\mathbf{1}_{m^{\prime}}
\end{array}\right] \leq\left[\begin{array}{cc}
\left(\mathbf{T}_{\mathrm{O}} \mathbf{G} \mathbf{T}_{\mathrm{I}}\right) & \left(\mathbf{T}_{\mathrm{O}} \mathbf{G} \overline{\mathbf{T}}_{\mathrm{I}}\right) \\
\left(\overline{\mathbf{T}}_{\mathrm{O}} \mathbf{G} \mathbf{T}_{\mathrm{I}}\right) & \left(\overline{\mathbf{T}}_{\mathrm{O}} \mathbf{G} \overline{\mathbf{T}}_{\mathrm{I}}\right)
\end{array}\right]\left[\begin{array}{c}
\mathbf{T}_{\mathrm{I}}^{T} \\
\overline{\mathbf{T}}_{\mathrm{I}}^{T}
\end{array}\right] \mathbf{u}_{i}^{\mathrm{c}}-\mathbf{P} \mathbf{v}_{i} \leq M\left[\begin{array}{c}
\mathbf{0}_{q} \\
\mathbf{1}_{m^{\prime}}
\end{array}\right]
$$

Because set point changes are considered for controlled variables only, the big-M eq. (12d) is used to force the trivial solution $\mathbf{u}_{i}^{\mathrm{c}}=\mathbf{0}_{n}$ for each uncontrolled variable $i$ (i.e., whenever $\left.\mathbf{z}^{\mathrm{O}}(i)=0\right)$. Otherwise, we can assume that $i \in \mathrm{CVs}$ and if eq. (12e) is pre-multiplied by $\mathbf{Q}^{T}$ we have:

$$
-M\left[\begin{array}{c}
\mathbf{1}_{q} \\
\mathbf{0}_{n^{\prime}}
\end{array}\right] \leq\left[\begin{array}{c}
\mathbf{T}_{\mathrm{I}}^{T} \\
\overline{\mathbf{T}}_{\mathrm{I}}^{T}
\end{array}\right] \mathbf{u}_{i}^{\mathrm{c}} \leq M\left[\begin{array}{c}
\mathbf{1}_{q} \\
\mathbf{0}_{n^{\prime}}
\end{array}\right]
$$

Recall that eqs. (16) and (17) are equivalent to eqs. (12b) and (12e), respectively, when specific CVs and MVs are selected by fixing the values of $\mathbf{z}^{\mathrm{O}}$ and $\mathbf{z}^{\mathrm{I}}$. In eq. (17), assuming that the output $i$ is being controlled, the variables of $\mathbf{u}_{i}^{\mathrm{c}}$ that are not selected as MVs are 
driven to zero by the big-M conditions. In turn, because of these zero entries of $\mathbf{u}_{i}^{\mathrm{c}}$, only the subsystems given by $\mathbf{G}_{\mathrm{s}}=\mathbf{T}_{\mathrm{O}} \mathbf{G} \mathbf{T}_{\mathrm{I}}$ and $\mathbf{G}_{\mathrm{r}}=\overline{\mathbf{T}}_{\mathrm{O}} \mathbf{G} \mathbf{T}_{\mathrm{I}}$ (refer also to eqs. (4g) and (4h)) are taken into account in eq. (16). In this big-M constraint, after the transformation $\mathbf{P}$ is applied, both the lower and upper bounds of the first $q$ rows are driven to zero. Thus, eq. (16) guarantees that the set point change conditions given in eq. (13) hold for each controlled variable $i$.

Finally, if the same procedure applied to eq. (16) is also used in eq. (12g), then eq. (18) is obtained.

$$
\mathbf{P} \mathbf{y}_{i}^{\mathrm{c}}=\left[\begin{array}{cc}
\left(\mathbf{T}_{\mathrm{O}} \mathbf{G} \mathbf{T}_{\mathrm{I}}\right) & \left(\mathbf{T}_{\mathrm{O}} \mathbf{G} \overline{\mathbf{T}}_{\mathrm{I}}\right) \\
\left(\overline{\mathbf{T}}_{\mathrm{O}} \mathbf{G} \mathbf{T}_{\mathrm{I}}\right) & \left(\overline{\mathbf{T}}_{\mathrm{O}} \mathbf{G} \overline{\mathbf{T}}_{\mathrm{I}}\right)
\end{array}\right]\left[\begin{array}{c}
\mathbf{T}_{\mathrm{I}}^{T} \\
\overline{\mathbf{T}}_{\mathrm{I}}^{T}
\end{array}\right] \mathbf{u}_{i}^{\mathrm{c}}-\mathbf{P}\left(\mathbf{v}_{i} \otimes \mathbf{z}^{\mathrm{O}}\right)
$$

In eq. (18), the term $\mathbf{P}\left(\mathbf{v}_{i} \otimes \mathbf{z}^{\mathrm{O}}\right)$ is either equal to $\mathbf{P} \mathbf{v}_{i}$ if $i$ is a selected controlled variable (i.e., $\mathbf{z}^{\mathrm{O}}(i)=1$ ) or equal to $\mathbf{0}_{m}$ otherwise. In the former case, since the right hand sides of the first $q$ rows of eq. (18) are the same than the corresponding constrained values of the first rows of eq. (16), the first $q$ entries of the vector $\mathbf{P} \mathbf{y}_{i}^{\mathbf{c}}$ are zero. In eq. (16), the big-M conditions of the remaining rows are relaxed and, therefore, $\mathbf{y}_{i}^{\mathrm{c}}$ will only have nonzero values associated to the uncontrolled variables (UVs). In the latter case, because eq. (12d) forced the condition $\mathbf{u}_{i}^{\mathrm{c}}=\mathbf{0}_{n}$, then the trivial solution $\mathbf{y}_{i}^{\mathrm{c}}=\mathbf{0}_{m}$ is obtained if $i \notin \mathrm{CVs}$.

A similar argument can be applied to eqs. (12c), (12f), and (12h) to demonstrate that $\mathbf{y}_{j}^{\mathrm{d}}$ and $\mathbf{u}_{j}^{\mathrm{d}}$ represent the solution of eq. (13) for the $j$-th disturbance change. In this case, the formulation is simpler because every disturbance is considered. The remaining constraints, eqs. (12i) and (12j), guarantee that the number of CVs and MVs are the same (square control structure) and the selected number of control loops is lower or equal than $q^{\max }$. It is important to note that $q^{\max }$ can be selected by the user from the range $1 \leq q^{\max } \leq \min (m, n)$. When $q^{\max }=\min (m, n)$, eq. $(12 \mathrm{j})$ is redundant.

Let us consider a generic system $\mathbf{y}_{i}=\operatorname{Ar}_{i}$ where $\mathbf{y}_{i}$ is the vector of deviations associated to a pre-defined $i$-th unitary change in $\mathbf{r}$ (i.e., the vector $\mathbf{r}_{i}$ has a unitary entry in the position 
$i$ and zero elsewhere). Then, the sum of squared deviations for all the variables considered individually results $\sum_{i=1}^{m}\left\|\mathbf{y}_{i}\right\|_{2}^{2}=\operatorname{tr}\left(\mathbf{A}^{T} \mathbf{A}\right)=\|\mathbf{A}\|_{F}^{2}$. In order to calculate the SSD index, recall that from eq. (5) it follows that:

$$
\mathbf{y}_{\mathrm{r}}^{\mathrm{sp}}=\left(\mathrm{G}_{\mathrm{r}} \mathrm{G}_{\mathrm{s}}^{-1}\right) \mathbf{y}_{\mathrm{s}}^{\mathrm{sp}}, \quad \mathbf{y}_{\mathrm{r}}^{\mathrm{d}}=\left(\mathbf{D}_{\mathrm{r}}-\mathrm{G}_{\mathrm{r}} \mathbf{G}_{\mathrm{s}}^{-1} \mathbf{D}_{\mathrm{s}}\right) \mathbf{d}^{*}
$$

Therefore, for a given selection of CVs and MVs the solution of eqs. (12b) to (12h) includes multiple valid solutions of eq. (19). On the one hand, $\mathbf{y}_{\mathrm{r}}^{\mathrm{sp}}=\overline{\mathbf{T}}_{\mathrm{O}} \mathbf{y}_{i}^{\mathrm{c}}$ and $\mathbf{y}_{\mathrm{s}}^{\mathrm{sp}}=\mathbf{T}_{\mathrm{O}} \mathbf{v}_{i}$ solve eq. (19) for each $i$. On the other hand, $\mathbf{y}_{\mathrm{r}}^{\mathrm{d}}=\overline{\mathbf{T}}_{\mathrm{O}} \mathbf{y}_{j}^{\mathrm{d}}$ and $\mathbf{d}^{*}=\mathbf{v}_{j}$ are applied for each $j$. Therefore, the SSD index in eq. (4b) is equivalent to $\sum_{i=1}^{m}\left\|\mathbf{y}_{i}^{\mathrm{c}}\right\|_{2}^{2}+\sum_{j=1}^{p}\left\|\mathbf{y}_{j}^{\mathrm{d}}\right\|_{2}^{2}$, which in turn is the same that the first two components of the objective function given in eq. (12a).

\subsection{The NLE subproblem}

The controller structure design based on the NLE index, which has been described in Section 3.2 , is modeled here by means of the linear constraints in eqs. (12k) to (12r). Following the same strategy of previous section, the matrix partitioning defined in eqs. (4f) to (4h), the nonlinear Hadamard product in eq. (4i), and the nonlinear matrix operations in eq. (4c) are all avoided. If set-points and disturbances are considered separately, the following systems of equations derived from eq. (10) are used:

Set point changes: $\left[\begin{array}{l}\mathbf{y}_{i}^{\mathrm{nc}}=\mathbf{v}_{i}-\tilde{\mathbf{G}} \mathbf{u}_{i}^{\mathrm{c}} \\ \forall i \in \mathrm{CVs}\end{array}\right.$, Disturbance changes: $\left[\begin{array}{l}\mathbf{y}_{j}^{\mathrm{nd}}=-\tilde{\mathbf{G}} \mathbf{u}_{j}^{\mathrm{d}} \\ \forall j=1, \ldots, p\end{array}\right.$

In eq. (20), the matrix $\tilde{\mathbf{G}}$ will only include the entries of $\mathbf{G}$ selected for the controller structure. To choose particular entries of $\mathbf{G}$, the matrix of binary variables $\mathbf{z}^{\mathrm{n}}$ of size $(m \times n)$ is used. Since adding the definition $\tilde{\mathbf{G}}=\left(\mathbf{G} \otimes \mathbf{z}^{\mathrm{n}}\right)$ will introduce equations with bilinear terms between continuous decision variables to the model, a different approach is considered here. For instance, in eq. (20) the product between $\tilde{\mathbf{G}}$ and $\mathbf{u}_{i}^{\mathrm{c}}$ can be calculated as $\tilde{\mathbf{G}} \mathbf{u}_{i}^{\mathrm{c}}=$ 
$\left(\mathbf{G} \otimes \mathbf{z}^{\mathrm{n}}\right) \mathbf{u}_{i}^{\mathrm{c}}=\left(\mathbf{G} \otimes\left[\mathbf{z}^{\mathrm{n}} \otimes \mathbf{1}_{m}\left(\mathbf{u}_{i}^{\mathrm{c}}\right)^{T}\right]\right) \mathbf{1}_{n}$, where the term in square brackets can be obtained using (linear) big-M constraints. Also, an identical argument can be applied to the product $\tilde{\mathbf{G}} \mathbf{u}_{j}^{\mathrm{d}}$.

Based on the above discussion, new continuous variable matrices $\mathbf{u}_{i}^{\text {nc }}$ and $\mathbf{u}_{j}^{\text {nd }}$ are defined. The big-M inequalities given in eqs. (12k) to $(12 \mathrm{n})$ define the input response associated to the controller structure selected in $\mathbf{z}^{\mathrm{n}}$ for each type of change (i.e., set point or disturbance). If $\mathbf{z}^{\mathrm{n}}(k, l)=1$, because of eqs. (12k) and (12l), then $\mathbf{u}_{i}^{\mathrm{nc}}(k, l)=\mathbf{u}_{i}^{\mathrm{c}}(l), \forall i$, and $\mathbf{u}_{j}^{\mathrm{nd}}(k, l)=$ $\mathbf{u}_{j}^{\mathrm{c}}(l), \forall j$. Otherwise, $\mathbf{z}^{\mathrm{n}}(k, l)=0$ and both $\mathbf{u}_{i}^{\mathrm{nc}}(k, l)$ and $\mathbf{u}_{j}^{\mathrm{nd}}(k, l)$ are driven to zero by eqs. (12m) and (12n). As a result, $\mathbf{u}_{i}^{\mathrm{nc}}=\left[\mathbf{z}^{\mathrm{n}} \otimes \mathbf{1}_{m}\left(\mathbf{u}_{i}^{\mathrm{c}}\right)^{T}\right]$ and $\mathbf{u}_{j}^{\mathrm{nd}}=\left[\mathbf{z}^{\mathrm{n}} \otimes \mathbf{1}_{m}\left(\mathbf{u}_{j}^{\mathrm{d}}\right)^{T}\right]$.

The corresponding output deviations vectors $\mathbf{y}_{i}^{\mathrm{nc}}$ and $\mathbf{y}_{j}^{\text {nd }}$ (associated to the $i$-th setpoint and $j$-th disturbance change, respectively) are calculated by eqs. (12o) and (12p). The constraints in eqs. (12q) and (12r) are structural constraints. They guarantee that the controller structure $\mathbf{z}^{\mathrm{n}}$ has only entries in allowed positions defined by the selection of CVs and MVs given by $\mathbf{z}^{\mathrm{O}}$ and $\mathbf{z}^{\mathrm{I}}$, respectively, and ensure that at least a decentralized policy is used. Finally, similar to previous section, the NLE index (last two components of eq. (12a)) can be computed as $\sum_{i=1}^{m}\left\|\mathbf{y}_{i}^{\mathrm{nc}}\right\|_{2}^{2}+\sum_{j=1}^{p}\left\|\mathbf{y}_{j}^{\mathrm{nd}}\right\|_{2}^{2}$

\subsubsection{RGA-based input-output pairing}

The input-output pairing problem is modeled here using eqs. (12s) to (12v). The main idea is to represent the selection of the decentralized control structure with a new matrix of binary decision variables $\mathbf{z}^{\text {nd }}$, which will be linked to the sparse control structure selection $\mathbf{z}^{\mathrm{n}}$. This approach has two main objectives: 1- to serve as a base design platform, around which the sparse structure is defined and 2- to define the input-output pairing by using the so-called relative gain array (RGA), $\Lambda=\mathbf{G}_{\mathrm{s}} \otimes\left(\mathbf{G}_{\mathrm{s}}^{-1}\right)^{T}$, where $\mathbf{G}_{\mathrm{s}}$ is the selected process to be controlled. In other words, the unitary entries in $\mathbf{z}^{\text {nd }}$ correspond to proper and feasible gains in $\Lambda$.

The binary variables $\mathbf{z}^{\text {nd }}$ are constrained by eqs. (12s) to (12t). Equation (12s) defines 
two structural feasibility constraints, which guarantee a one-to-one pairing between MVs and CVs. Besides, eq. (12t) guarantees that the decentralized control structure will be the base case scenario to perform the sparse control design.

In order to compute the RGA while avoiding the introduction of strong non-linearities in the model, it is clear that some kind of reformulation is needed. According to the precursory work of Bristol ${ }^{6}$, each element of the matrix $\Lambda$ is defined as $\lambda_{i l}=g_{i l} \hat{g}_{l i}$, where

$$
g_{i l}=\left.\frac{\Delta y_{i}}{\Delta u_{l}}\right|_{u_{k}=0 \forall k \neq l, u_{l}: \text { free }} \quad \text { and } \quad \hat{g}_{l i}=\left.\frac{\Delta u_{l}}{\Delta y_{i}}\right|_{y_{k}=0 \forall k \neq i, y_{i}: \text { free }}
$$

represent the open-loop gains and $1 / \hat{g}_{l i}$ the closed-loop gains of the process $\mathbf{G}_{\mathrm{s}}$ at steadystate. Let us consider a generic process $\mathbf{G}$ of $(m \times n)$ with the following open-loop gain matrix:

$$
\mathbf{G}=\left[\begin{array}{cccc}
g_{11} & g_{12} & \cdots & g_{1 n} \\
g_{21} & g_{22} & \cdots & g_{2 n} \\
\vdots & \vdots & \ddots & \vdots \\
g_{m 1} & g_{m 2} & \cdots & g_{m n}
\end{array}\right]=\left[\begin{array}{cccc}
\Delta y_{1} / \Delta u_{1} & \Delta y_{1} / \Delta u_{2} & \cdots & \Delta y_{1} / \Delta u_{n} \\
\Delta y_{2} / \Delta u_{1} & \Delta y_{2} / \Delta u_{2} & \cdots & \Delta y_{2} / \Delta u_{n} \\
\vdots & \vdots & \ddots & \vdots \\
\Delta y_{m} / \Delta u_{1} & \Delta y_{m} / \Delta u_{2} & \cdots & \Delta y_{m} / \Delta u_{n}
\end{array}\right]
$$

where the $l$-th column is computed based on the condition $\Delta u_{k}=0, \forall k \neq l$. Similarly, the closed-loop gain can be computed as,

$$
\hat{\mathbf{G}}=\left[\begin{array}{cccc}
\hat{g}_{11} & \hat{g}_{12} & \cdots & \hat{g}_{1 m} \\
\hat{g}_{21} & \hat{g}_{22} & \cdots & \hat{g}_{2 m} \\
\vdots & \vdots & \ddots & \vdots \\
\hat{g}_{n 1} & \hat{g}_{n 2} & \cdots & \hat{g}_{n m}
\end{array}\right]=\left[\begin{array}{cccc}
\Delta u_{1} / \Delta y_{1} & \Delta u_{1} / \Delta y_{2} & \cdots & \Delta u_{1} / \Delta y_{m} \\
\Delta u_{2} / \Delta y_{1} & \Delta u_{2} / \Delta y_{2} & \cdots & \Delta u_{2} / \Delta y_{m} \\
\vdots & \vdots & \ddots & \vdots \\
\Delta u_{n} / \Delta y_{1} & \Delta u_{n} / \Delta y_{2} & \cdots & \Delta u_{n} / \Delta y_{m}
\end{array}\right]
$$

where the $i$-th column is computed based on the condition $\Delta y_{k}=0, \forall k \neq i$. Thus, it is clear that $\Lambda=\mathbf{G} \otimes \hat{\mathbf{G}}^{T}$.

Considering the closed-loop gain definition in eq. (21), we have $\Delta u_{l}=\left.\hat{g}_{l i} \Delta y_{i}\right|_{y_{k}=0 \forall k \neq i, y_{i} \text { : free }}$. 
Furthermore, if we consider unitary step changes for $\Delta y_{i}$ (like in Section 4.1) the columns in eq. (23) become

$$
\left[\begin{array}{cccc}
\Delta u_{1}^{\mathrm{c}}(1) & \Delta u_{2}^{\mathrm{c}}(1) & \cdots & \Delta u_{m}^{\mathrm{c}}(1) \\
\Delta u_{1}^{\mathrm{c}}(2) & \Delta u_{2}^{\mathrm{c}}(2) & \cdots & \Delta u_{m}^{\mathrm{c}}(2) \\
\vdots & \vdots & \ddots & \vdots \\
\Delta u_{1}^{\mathrm{c}}(n) & \Delta u_{2}^{\mathrm{c}}(n) & \cdots & \Delta u_{m}^{\mathrm{c}}(n)
\end{array}\right]=\left[\mathbf{u}_{1}^{\mathrm{c}}, \mathbf{u}_{2}^{\mathrm{c}}, \ldots, \mathbf{u}_{m}^{\mathrm{c}}\right]
$$

being $\mathbf{u}_{i}^{\mathrm{c}}$ with $i=1, \ldots, m$ the input deviation vectors previously defined for the SSD

approach. Hence, the RGA matrix can be computed as $\Lambda=\mathbf{G} \otimes\left[\mathbf{u}_{1}^{\mathrm{c}}, \mathbf{u}_{2}^{\mathrm{c}}, \ldots, \mathbf{u}_{m}^{\mathrm{c}}\right]^{T}$ where $\mathbf{G}$ is a fixed real matrix and $\otimes$ the Hadamard product (element-by-element product).

Taking into account the above discussion, the RGA evaluation is performed in eq. (12u) using big-M constraints based on the decentralized control structure selection defined by $\mathbf{z}^{\text {nd }}$. Matrix $\mathbf{y}^{\mathrm{r}}$ includes both the selected entries of the RGA, which are constrained by eq. (12v) guaranteeing a feasible and useful input-output pairing according to the scalar parameters $\delta_{1}$ and $\delta_{2}$ fixed by the user, and the entries not selected (i.e., those associated to zeros in $\left.\mathbf{z}^{\text {nd }}\right)$ which are also driven to zero by eq. (12v).

\subsubsection{Stability/robustness criterion}

The stability/robustness criterion shown in eq. (4j) is a well known steady-state test used to design the structure of multivariable IMC controllers, specially in the sparse control case. As is, this eigenvalue-based test introduces hard non-linearities in the model, and some kind of reformulation is needed. In order to obtain this reformulation, the matrix properties to be used are revisited first.

Any real matrix $\mathbf{A}$ of size $(n \times n)$ is called a positive stable $(\mathrm{PS})$ matrix if $\operatorname{Re}\left[\lambda_{i}(\mathbf{A})\right]>$ $0, \forall i=1, \ldots, n$, where $\lambda_{i}(\cdot)$ calculates the $i$-th eigenvalue and $\operatorname{Re}(\cdot)$ is the real part function. Moreover, if $\mathbf{A}$ is PS then $\mathbf{A}^{-1}$ is also a PS matrix ${ }^{26}$. Additionally, since any square real matrix has only real or complex conjugate eigenvalues, for any real PS matrix $\mathbf{A}$ the following 
trace condition is true,

$$
\operatorname{tr}(\mathbf{A})=\sum_{i=1}^{n} \lambda_{i}(\mathbf{A})=\sum_{i=1}^{n} \operatorname{Re}\left[\lambda_{i}(\mathbf{A})\right]=\sum_{i=1}^{n} a_{i i}>0
$$

where $a_{i j}$ is the $(i, j)$-entry in the matrix $\mathbf{A}$. It is important to note that the converse is not true, i.e., a positive trace does not imply PS. However, a negative trace implies that the matrix $\mathbf{A}$ is definitively not PS, and this fact is used as a preliminary stability/robustness test in the proposed MIQP model.

Let us consider the product $\mathbf{G}_{\mathrm{s}}\left(\tilde{\mathbf{G}}_{\mathbf{S}}\right)^{-1}$ and its inverse $\tilde{\mathbf{G}}_{\mathbf{s}}\left(\mathbf{G}_{\mathrm{S}}\right)^{-1}$, for a $(q \times q)$ process $\mathbf{G}_{\mathrm{s}}$. The trace of the latter matrix product can be written as:

$$
\begin{aligned}
\operatorname{tr}\left(\tilde{\mathbf{G}}_{\mathrm{s}}\left(\mathbf{G}_{\mathrm{s}}\right)^{-1}\right)= & \mathbf{r}_{1}^{T}\left(\tilde{\mathbf{G}}_{\mathrm{s}}\left(\mathbf{G}_{\mathrm{s}}\right)^{-1}\right) \mathbf{r}_{1}+\mathbf{r}_{2}^{T}\left(\tilde{\mathbf{G}}_{\mathrm{s}}\left(\mathbf{G}_{\mathrm{s}}\right)^{-1}\right) \mathbf{r}_{2}+\ldots \\
& +\mathbf{r}_{q}^{T}\left(\tilde{\mathbf{G}}_{\mathrm{s}}\left(\mathbf{G}_{\mathrm{s}}\right)^{-1}\right) \mathbf{r}_{q}
\end{aligned}
$$

where the vector $\mathbf{r}_{k} \in \mathbb{R}^{q}$ is a column vector with a unitary entry in the position $k$ and zero elsewhere. In order to evaluate the trace in eq. (26), recall that the formulation in eq. (12) is defined to work with non square processes $\mathbf{G}$ of $(m \times n)$, where the selection of the square subprocess $\mathbf{G}_{\mathrm{S}}($ of size $q \times q)$ to be controlled is determined by the binary vectors $\mathbf{z}^{\mathrm{O}}$ and $\mathbf{z}^{\mathrm{I}}$. Moreover, $\tilde{\mathbf{G}}_{\mathrm{S}}$ is a particular selection of entries of $\mathbf{G}_{\mathrm{s}}$.

Taking into account the transformation matrix $\mathbf{T}_{\mathrm{O}}$ of size $(q \times m)$, for each $k$ there is a unique unit vector $\mathbf{v}_{i}$ such that $\mathbf{r}_{k}=\mathbf{T}_{\mathrm{O}} \mathbf{v}_{i}$. Since from eqs. (16) and (17) it follows that $\mathbf{T}_{\mathrm{I}}^{T} \mathbf{u}_{i}^{\mathrm{c}}=\left(\mathbf{G}_{\mathrm{s}}\right)^{-1} \mathbf{T}_{\mathrm{O}} \mathbf{v}_{i}$, and given that $\mathbf{G}_{\mathrm{s}}=\mathbf{T}_{\mathrm{O}} \mathbf{G} \mathbf{T}_{\mathrm{I}}$ and $\tilde{\mathbf{G}}_{\mathrm{s}}=\mathbf{T}_{\mathrm{O}} \tilde{\mathbf{G}} \mathbf{T}_{\mathrm{I}}$, then eq. (26) becomes:

$$
\begin{aligned}
\operatorname{tr}\left(\tilde{\mathbf{G}}_{\mathrm{S}}\left(\mathbf{G}_{\mathrm{S}}\right)^{-1}\right) & =\sum_{i=1}^{m}\left(\mathbf{T}_{\mathrm{O}} \mathbf{v}_{i}\right)^{T} \tilde{\mathbf{G}}_{\mathrm{s}}\left(\mathbf{G}_{\mathrm{S}}\right)^{-1}\left(\mathbf{T}_{\mathrm{O}} \mathbf{v}_{i}\right) \\
& =\sum_{i=1}^{m} \mathbf{v}_{i}^{T}\left(\mathbf{T}_{\mathrm{O}}^{T} \tilde{\mathbf{G}}_{\mathrm{S}} \mathbf{T}_{\mathrm{I}}^{T}\right) \mathbf{u}_{i}^{\mathrm{c}} \\
& =\sum_{i=1}^{m} \mathbf{v}_{i}^{T} \tilde{\mathbf{G}} \mathbf{u}_{i}^{\mathrm{c}}
\end{aligned}
$$


Moreover, if the set point changes in eq. (20) are considered, the constraint in eq. (12o) implies that $\tilde{\mathbf{G}} \mathbf{u}_{i}^{\mathrm{c}}=\left(\mathbf{v}_{i} \otimes \mathbf{z}^{\mathrm{O}}\right)-\mathbf{y}_{i}^{\mathrm{nc}}$ and eq. (28) results:

$$
\operatorname{tr}\left(\tilde{\mathbf{G}}_{\mathrm{S}}\left(\mathbf{G}_{\mathrm{s}}\right)^{-1}\right)=\sum_{i=1}^{m} \mathbf{v}_{i}^{T}\left(\left(\mathbf{v}_{i} \otimes \mathbf{z}^{\mathrm{O}}\right)-\mathbf{y}_{i}^{\mathrm{nc}}\right)=\sum_{i=1}^{m}\left(\mathbf{z}^{\mathrm{O}}(i)-\mathbf{y}_{i}^{\mathrm{nc}}(i)\right)
$$

where at the last step the definition of column vectors $\mathbf{v}_{i}$ has been applied.

Note that eq. (28) evaluates the trace of the matrix $\mathbf{G}_{\mathrm{S}}\left(\tilde{\mathbf{G}}_{\mathrm{S}}\right)^{-1}$ for any square subprocess $\mathbf{G}_{\mathrm{S}}$ selected by the model. Hence, an alternative stability/robustness test to eq. (4j) is included in the formulation in eq. $(12 \mathrm{w})$, where a small positive lower bound $\delta$ is considered. This conservative constraint avoids the selection of control structures which are definitely not PS. It is important to recall, however, that a positive trace is a necessary but not sufficient condition to guarantee the PS property of the matrix.

Note 2: An alternative approach to implement the stability/robustness criterion is to combine the proposed model with an iterative procedure based on testing the stability, adding cuts, and rerunning. For instance, after using GAMS/CPLEX to solve the formulation given in eq. (12), the best solution obtained can be transferred to an environment such as Matlab to perform the stability/robustness test stated in eq. (4j) and, in case of instability, add a constraint (cut) to the formulation in eq. (12). The added constraint discards from the search space of the MIQP problem the binary solution resulting in an unstable controller structure, and the augmented formulation is rerun at the following iteration. The overall procedure is continued until a stable/robust solution is found. This methodology has not been required in this work because the solutions found in the test cases were all stable.

\subsection{Heuristics and controller structure}

Heuristic considerations selecting any particular multivariable control structure design can be easily incorporated to the formulation in eq. (12). For example, if the $i$-th output variable and the $l$-th input variable are selected to be ad hoc controlled and manipulated variables, 
respectively, then this situation can be considered by fixing the value of the corresponding binary variables, i.e., $\mathbf{z}^{\mathrm{O}}(i)=1$ and $\mathbf{z}^{\mathrm{I}}(l)=1$. These ad-hoc decisions are usually determined before the control structure design procedure is applied, e.g., decisions from the process synthesis stage, good practices from practitioners or process engineers, and/or safety issues. Furthermore, different controller topologies (i.e., control loop architectures), such as decentralized, sparse, and full, can be designed by the user. The model in eq. (12) can be regarded as a general formulation to design sparse controllers, i.e., controllers with arbitrary interaction among their loops. Depending on the size of the control structure selected, $q=\left\|\mathbf{z}^{\mathrm{O}}\right\|=\left\|\mathbf{z}^{\mathrm{I}}\right\|$, these type of controllers can be implemented either by using IMC and/or MPC theory or via multiple decentralized interacting PIDs. However, also decentralized and full controllers can be designed. On the one hand, a decentralized non-interacting (diagonal) controller is characterized by several PID control loops which have no interaction among them. This type of controller can be designed with the proposed method if the equality $\mathbf{z}^{\mathrm{n}}=\mathbf{z}^{\text {nd }}$ replaces the inequality in eq. (12t). On the other hand, a full controller means that a complete interaction among the control loops is required, and this design context is considered if the inequality $\mathbf{z}^{\mathrm{n}}(i, l) \geq \mathbf{z}^{\mathrm{I}}(l)+\mathbf{z}^{\mathrm{O}}(i)-1$ is added to the model (i.e., no plant-model mismatch is forced).

\subsection{General comments of the MIQP-based MCS design approach}

\subsubsection{The Big-M representation}

The big-M method is a well-know modeling technique used to formulate constraints that apply only when certain conditions over the binary variables hold. When big-M constraints are used to represent disjunctions, the convergence rate of the solver and amount of computational resources needed are, in many cases, sensitive to the selected $M$ parameter. In the proposed MIQP method, the same sufficiently large value of $M$ is used in all constraints. An alternative approach will consider the selection of an individual $M_{c}$ for each constraint $c$, such that $M_{c}$ is as small as possible. Hence, the resulting formulation will be as tight as possible. 
However, in the context of the proposed MIQP framework the calculation of these $M_{c}$ is not straightforward. Indeed, a rigorous approach requires solving multiple mixed-integer optimization problems. As an example, sharp big-M parameters for eq. (12b) can be obtained by calculating the minimum and maximum values of each component of $\mathbf{y}_{i}^{\mathrm{c}}$ considering every integer solution (i.e., every alternative controller structure) such that the corresponding entry of $\mathbf{z}^{\mathrm{O}}$ is set to zero. This analysis is out of the scope of the present contribution. In order to give some insight on the sensitivity to the value of $M$ of the proposed MIQP approach, results for alternative values of $M$ are reported for one of the case studies in the Supporting Information file.

\subsubsection{The RGA interaction}

The classical RGA-based input-output pairing selects particular entries of the matrix $\Lambda=$ $\mathbf{G}_{\mathrm{s}} \otimes\left(\mathbf{G}_{\mathrm{s}}^{-1}\right)^{T}$ which feature predefined/desired values, where $\mathbf{G}_{\mathrm{s}}$ is the steady-state gain matrix of the process to be controlled. The main and well-known drawback of this approach is the loss of reliability when the process $\mathbf{G}_{\mathrm{s}}$ is ill-conditioned or almost singular.

The proposed MIQP approach for MCS design avoids this drawback because of the inherent properties of the SSD and NLE indexes discussed in Sections 3.1 and 3.2, respectively. On the one hand, the SSD minimization tends to maximize the minimum singular value of the process $\mathbf{G}_{\mathrm{s}}$, i.e. a well conditioned process and reliable RGA is obtained. On the other hand, the NLE minimization drives the selection of the input-output pairing based on minimizing the net load effect in eq. (10), i.e. the multivariable interaction at closed loop is minimized ${ }^{12,24}$. In this context, the inequality constraints in eq. (12v) become a simple feasibility test to guarantee an interaction in the range $\left[\delta_{1}, \delta_{2}\right]$, which is fixed by the user. It is worth noting that, while $\delta_{1}$ represents the most important limit, i.e. the minimum acceptable interaction, the upper limit $\delta_{2}$ can be set large enough because the interaction tends to be minimized by the SSD+NLE combined index. Since the attainable RGA interaction depends on the characteristics of the process itself, some particular intervals for the range 
$\left[\delta_{1}, \delta_{2}\right]$ can turn the MIQP problem infeasible. For instance, if the lower limit $\delta_{1}$ is set too high, solutions may not be available for some predefined number $q^{\max }$ of required control loops. A similar situation may happen if the range $\left[\delta_{1}, \delta_{2}\right]$ is fixed to be extremely small. The definition of alternative interaction ranges is analyzed for one of the case studies in the Supporting Information file.

It is important to note that the BMINLP formulation in eq. (4) depends on the RGAnumber, but the single-level MIQP reformulation presented in eq. (12) only depends on the RGA. The RGA-number for input-output pairing is avoided based on the arguments explained in Section 3.3. At the BMINLP formulation level, the inner optimization in eq. $(4 \mathrm{~m})$ based on the RGA-number can be replaced by any alternative methodology for input-output pairing that selects a single solution from a set of feasible solutions. In the same sense, the eqs. (12u)-(12v) of the MIQP model can be modified replacing the RGA constraints by an alternative input-output pairing criteria. For instance, the relative interaction array (RIA) could be used instead of the RGA method. In this case, since the RIA is based on the RGA elements, from the feasible pairing point of view it can be assumed that there is no strong difference between both methodologies. However, since the RIA elements are inversely proportional to the RGA ones, some kind of linearization/reformulation is needed to guarantee the linear property of the MIQP constraints.

\subsubsection{Strengths and weaknesses}

It is important to recall that the proposed SSD+NLE-based MIQP approach for control structure design is based on a linearized model of the process under study. Therefore, no dynamic implications are considered and only a steady-state model is required. Obviously, like any model-based control approach (RGA, IMC, MPC, etc.), the performance and confidence of the extended minimum squared deviations (MSD) methodology are bounded by the validity zone of the process model itself.

Although the proposed approach addresses all the major issues of plant-wide control 
design such as the selection of CVs and MVs, the pairing between these sets, the structure of the controller (decentralized, sparse, full), and the corresponding tuning (via the process model and the IMC criterion), some other important issues remain unattended. Examples of these issues are to incorporate: 1- the selection of stabilizing control loops to design MCS on unstable processes, 2- other control topologies (control loop architectures) such as cascade control and/or feedforward control, 3- economic considerations of the overall MCS design, and 4- dynamic information if available.

The main limitation of the NLE steady-state minimization relies on the strictly proper systems hypothesis, which is true for industrial process in general. The theoretical conclusions about the NLE approach are limited to the bandwidth in which the control is effective. If the process has multivariable gains (singular values) modifications at high frequency, the multivariable controller bandwidth needs to be modified to avoid these frequencies. In general, if dynamic information of the process is available, a frequency analysis of the sensitivity functions, the loop gain function and/or a robust performance/stability analysis might be useful to define the bandwidth properly as it is suggested by a previous work of the authors ${ }^{24}$.

\section{Case studies}

In this section the proposed MIQP formulation for MCS design is tested on two classical medium/large scale benchmark processes. The reader may also find a simple step-by-step application of the proposed method in the Supporting Information file.

\subsection{The Tennessee Eastman process}

The first case study selected to test the proposed formulation is the well-known Tennessee Eastman process (TEP). This plant was introduced by Downs and Vogel ${ }^{27}$ as a multivariable nonlinear benchmark for the process control community. The process consists of five major units: the reactor, the product condenser, a recycle compressor, a vapor-liquid separator, 
and the product stripper. The plant produces two liquid products $\mathrm{G}$ and $\mathrm{H}$ from four gaseous reactants called A, C, D, and E, where also present are an inert B and a byproduct F. The reactions are approximately first-order with respect to the reactant concentrations, irreversible, and exothermic.

The plant summarizes 12 available MVs, 41 potential measurements, 20 disturbance scenarios, and six potential operating modes which define several product $\mathrm{G} / \mathrm{H}$ mass ratios and rates. The most popular operating condition is the base case or mode I, where a 50/50 mass ratio is required with flowrates of $7038 \mathrm{~kg} / \mathrm{h}$ for both $\mathrm{G}$ and $\mathrm{H}$.

The TEP is an open-loop unstable plant, so a proper stabilizing control structure is needed before applying the approach introduced in previous sections. In this work, the stabilizing control policy proposed by McAvoy and $\mathrm{Ye}^{28}$ is adopted, which consists of flow (inner) and level (cascade) controllers for the reactor, separator, and stripper. Figure 1 shows the TEP layout and the corresponding stabilizing control structure.

The main scenario considered here for multivariable control structure design is shown in Table 1 and corresponds to the aforementioned base case operating point, with $m=12$ outputs, $n=8$ inputs, and $p=2$ disturbances. The same framework has been used in previous publications of one of the authors ${ }^{11,12}$. The original control requisites defined by Downs and $\operatorname{Vogel}^{27}$ require the output variables identified as $y_{9}, y_{10}, y_{11}$, and $y_{12}$ to be controlled, being this fact indicated with an asterisk in Table 1.

The methodology proposed in eq. (12) is applied to the TEP considering decentralized, sparse, and full controller designs, and the corresponding results are shown in Tables 2 to 5. According to Section 4.3, the original control requisites are fixed as $\mathbf{z}^{\mathrm{O}}(i)=1, \forall i=$ 9, 10, 11, 12. As discussed in Section 4.2.1, the range of feasible input-output pairing values selected is $\left[\delta_{1}, \delta_{2}\right]=[0.3,30]$. Besides, in all cases the big-M value is fixed to $M=30$.

In order to illustrate the complexity of the associated MIQP problems, Table 2 includes the model sizes and relaxed solutions obtained for each controller type. In Table 3 a comparison of solutions and computational times is presented for both the GA methodology based 


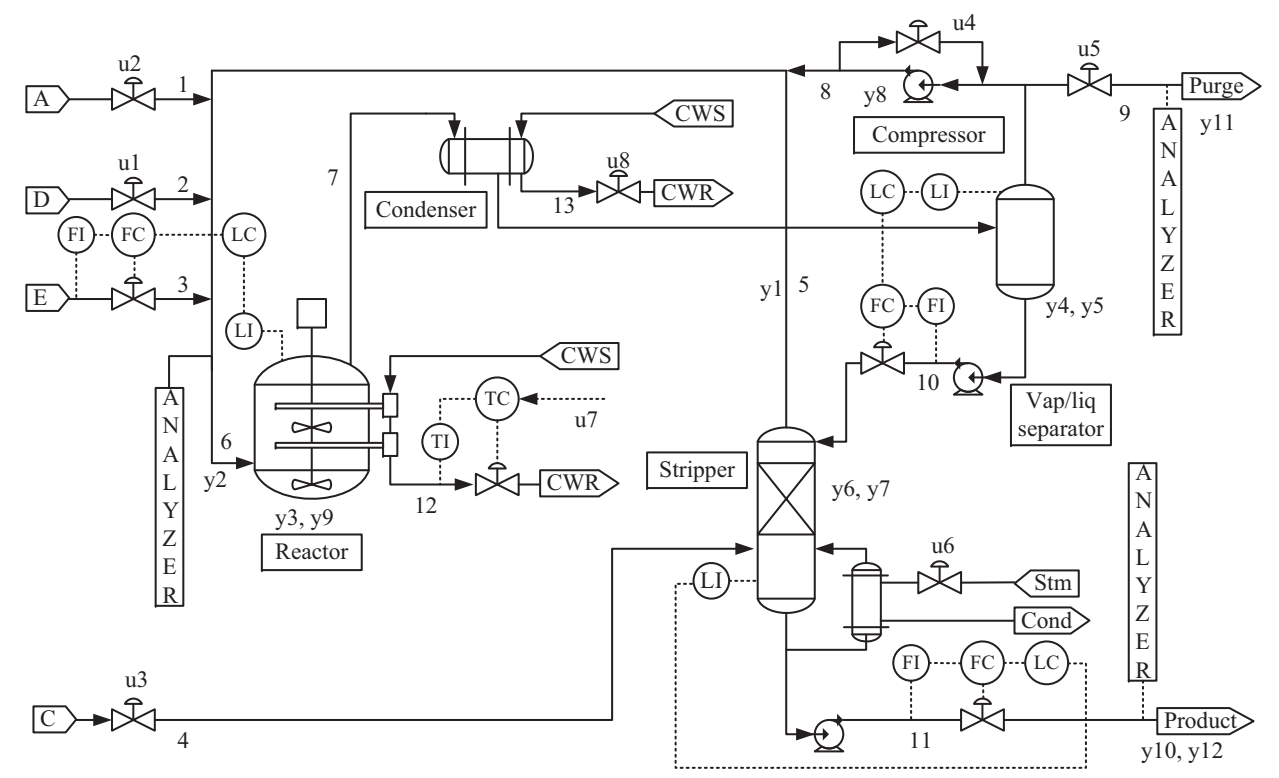

Figure 1: TEP with stabilizing control loops.

on the BMINLP formulation in eq. (4) and the proposed MIQP reformulation in eq. (12) solved with CPLEX in the GAMS context. All the simulations and optimizations were performed on an Intel@ i5 3.2GHz processor with 8GB of RAM, using Matlabß 2013 and GAMS v24.5.6/CPLEX 12.6.

While the GA procedure solves a more general bilevel problem, it is a stochastic global search approach that does not guarantee the optimality of the best solution found. In contrast, the proposed MIQP optimization model is applied to a simpler single-level problem and guarantees that the best solution obtained is also optimal whenever the solver's branching process is completed. Considering the results in Table 3, not only the MIQP solver has been able to complete the search process reporting the optimal solution for each controller type, but also the following two remarkable results can be observed: 1- the optimization times of the MIQP/CPLEX approach represent only 37.81\%, 47.84\%, and 5.36\% of the GA times for the controller designs called decentralized, sparse and full, respectively, and 2- for the sparse controller design, the GA-based BMINLP approach cannot find the optimal solution, getting the algorithm entrapped in some local minimum (it finishes by the function tolerance criterion, i.e., the fitness function improvement is less than certain tolerance.) These facts 
Table 1: TEP variables

\begin{tabular}{lll}
\hline & Inputs & Variable \\
\hline$u_{1}$ & D flow $[\mathrm{kg} / \mathrm{h}]$ & $X M V(1)$ \\
$u_{2}$ & A flow $[\mathrm{kg} / \mathrm{h}]$ & $X M V(3)$ \\
$u_{3}$ & A/C flow $[\mathrm{kscmh}]$ & $X M V(4)$ \\
$u_{4}$ & Compressor rec. valve [\%] & $X M V(5)$ \\
$u_{5}$ & Purge valve [\%] & $X M V(6)$ \\
$u_{6}$ & Stripper steam valve [\%] & $X M V(9)$ \\
$u_{7}$ & RCWO temp. setpoint $\left[{ }^{\circ} \mathrm{C}\right]$ & $X M E(21) s p$ \\
$u_{8}$ & CCW Flow $[\mathrm{m} 3 / \mathrm{h}]$ & $X M V(11)$ \\
\hline & Outputs & \\
\hline$y_{1}$ & Recycle flow $[\mathrm{kscmh}]$ & $X M E(5)$ \\
$y_{2}$ & Reactor flow $[\mathrm{kscmh}]$ & $X M E(6)$ \\
$y_{3}$ & Reactor temp. $\left[{ }^{\circ} \mathrm{C}\right]$ & $X M E(9)$ \\
$y_{4}$ & Separator temp. $\left[{ }^{\circ} \mathrm{C}\right]$ & $X M E(11)$ \\
$y_{5}$ & Separator pressure $[\mathrm{kPa}]$ & $X M E(13)$ \\
$y_{6}$ & Stripper pressure $[\mathrm{kPa}]$ & $X M E(16)$ \\
$y_{7}$ & Stripper temp. $\left[{ }^{\circ} \mathrm{C}\right]$ & $X M E(18)$ \\
$y_{8}$ & Compressor work $[\mathrm{kW}]$ & $X M E(20)$ \\
$y_{9}(*)$ & Reactor pressure $[\mathrm{kPa}]$ & $X M E(7)$ \\
$y_{10}(*)$ & Production rate $[\mathrm{m} 3 / \mathrm{h}]$ & $X M E(17)$ \\
$y_{11}(*)$ & B comp. purge $[\mathrm{mol} \%]$ & $X M E(30)$ \\
$y_{12}(*)$ & G/H comp. ratio & $X M E E_{G} / H$ \\
\hline & Disturbances & $I D V(1)$ \\
\hline$d_{1}$ & Composition stream 4 $(\mathrm{A} / \mathrm{C})$ & $I D V(2)$ \\
$d_{2}$ & Composition stream 4 $(\mathrm{B})$ & \\
\hline & &
\end{tabular}

Table 2: TEP results - MIQP model statistics for each type of controller design

\begin{tabular}{l|r|r|r}
\cline { 2 - 4 } & Decentralized & Sparse & Full \\
\hline Binary vars. & 112 & 208 & 208 \\
Continuous vars. & 2097 & 2097 & 2097 \\
Constraints & 10001 & 10350 & 10446 \\
Relaxed MIQP sol. & 2.899 & 2.899 & 2.899 \\
\hline
\end{tabular}

support the convenience of the proposed MIQP formulation.

Tables 4 and 5 show the optimal MCS designs obtained using the MIQP formulation for each controller type (decentralized, sparse, and full). On the one hand, Table 4 shows the CVs and MVs selected through the binary variable vectors $\mathbf{z}^{\mathrm{O}}$ and $\mathbf{z}^{\mathrm{I}}$, respectively. On the other hand, Table 5 displays the plant-model mismatch selection given by the unitary entries in $\mathbf{z}^{\mathrm{n}}$, the proposed input-output pairing defined by $\mathbf{z}^{\text {nd }}$ (which is highlighted with gray background), and the stability/robustness test performed off-line $\left(\min \left[\operatorname{Re}\left(\lambda_{i}\right)\right]\right)$. It is 
Table 3: TEP results - Comparison of GA-based BMINLP and MIQP/CPLEX solutions

\begin{tabular}{l|r|r|r|r|r|r}
\cline { 2 - 7 } & Decentralized & \multicolumn{2}{|c|}{ Sparse } & \multicolumn{2}{c}{ Full } \\
\cline { 2 - 7 } & GA & CPLEX & GA & CPLEX & GA & CPLEX \\
\hline Cost function (SSD+NLE) & 19.77 & 19.77 & 12.39 & 9.98 & 14.90 & 14.90 \\
Optimization time [s] & 484.85 & 183.32 & 48324.04 & 23115.89 & 278.28 & 14.92 \\
\hline
\end{tabular}

Table 4: TEP results - CVs and MVs selection

\begin{tabular}{|c|c|c|c|c|c|c|c|c|c|c|c|c|c|c|c|c|c|c|c|}
\hline & \multicolumn{11}{|c|}{$\mathbf{z}^{\mathrm{O}}$} & \multicolumn{8}{|c|}{$\mathbf{z}^{\mathrm{I}}$} \\
\hline & $y_{1}$ & $y_{2}$ & & & & & & & $19 y_{10}$ & $y_{11}$ & $y_{12}$ & $u_{1}$ & $u_{2}$ & $u_{3}$ & & $u_{5}$ & $u_{6}$ & & $u_{8}$ \\
\hline Decentralized & 0 & 1 & 0 & 0 & 0 & 0 & 1 & $\begin{array}{ll}0 & 1\end{array}$ & 11 & 1 & 1 & 1 & 1 & 1 & 0 & 1 & 0 & 1 & 1 \\
\hline Sparse & 1 & 0 & 0 & 0 & 0 & 0 & 1 & 1 & 1 & 1 & 1 & 1 & 1 & 1 & 1 & 1 & 1 & 1 & 0 \\
\hline Full & 1 & 0 & 0 & 0 & 0 & 0 & 0 & 1 & 1 & 1 & 1 & 1 & 1 & 1 & 1 & 1 & 0 & 1 & 0 \\
\hline
\end{tabular}

important to note that not only the number of CVs/MVs is different among decentralized, sparse, and full control policies, but also the variables being selected change. Indeed, the best decentralized control policy selected by the MIQP formulation in eq. (12) defines $y_{2}, y_{7}$, $y_{9}, y_{10}, y_{11}$, and $y_{12}$ to be the CVs and $u_{1}, u_{2}, u_{3}, u_{5}, u_{7}$, and $u_{8}$ as their MVs. In this case, a $(6 \times 6)$ diagonal control structure paired as shown in Table 5 , where $\mathbf{z}^{\mathrm{n}}=\mathbf{z}^{\text {nd }}$, is selected. Alternatively, the best sparse control structure defines a $(7 \times 7)$ interacting-multivariable control policy where $y_{1}, y_{7}, y_{8}, y_{9}, y_{10}, y_{11}, y_{12}$ and $u_{1}, u_{2}, u_{3}, u_{4}, u_{5}, u_{6}, u_{7}$ are selected as CVs and MVs, respectively. The degree of interaction for the controller is given by the unitary entries in Table 5, which represents the matrix $\mathbf{z}^{\mathrm{n}}$. Moreover, the corresponding input-output pairing is indicated by the highlighted entries with gray background, i.e. $\mathbf{z}^{\text {nd }}$. Finally, the best full control structure proposes manipulating the $u_{1}, u_{2}, u_{3}, u_{4}, u_{5}$, and $u_{7}$ input variables and controlling the $y_{1}, y_{8}, y_{9}, y_{10}, y_{11}$, and $y_{12}$ output variables. This structure represents a $(6 \times 6)$ multivariable control with complete interaction given by a $\mathbf{z}^{\mathrm{n}}$ matrix with full ones in the selected CVs and MVs. Table 5 also displays the corresponding input-output pairing in this case.

The optimal control structures obtained with the proposed MIQP model have been implemented and tested on the nonlinear dynamic TEP case study using Matlab. On the one hand, the best decentralized control structure has been implemented using multiple discrete PID-based control loops. On the other hand, both the sparse and full control structures 
Table 5: TEP results - Pairing $\left(\mathbf{z}^{\text {nd }}\right)$ and process-model mismatch $\left(\mathbf{z}^{\mathrm{n}}\right)$

\begin{tabular}{|c|c|c|c|c|c|c|c|c|c|c|c|c|c|c|c|c|c|c|c|c|c|}
\hline & \multicolumn{7}{|c|}{ Decentralized } & \multicolumn{7}{|c|}{ Sparse } & \multicolumn{7}{|c|}{ Full } \\
\hline & $u_{1}$ & $u_{2}$ & $u_{3} u_{4}$ & $u_{4} u_{5}$ & & & & $u_{1}$ & $u_{2} u$ & $u_{3} \quad u_{4}$ & & $u_{6}$ & & & $u_{1}$ & $u_{2}$ & & & $u_{6}$ & & $u_{8}$ \\
\hline$y_{1}$ & 0 & 0 & 0 & $\begin{array}{ll}0 & 0\end{array}$ & 0 & 0 & 0 & 1 & 0 & 1 & 1 & 0 & 1 & 0 & 1 & 1 & 1 & 1 & 0 & 1 & 0 \\
\hline$y_{2}$ & 0 & 0 & 0 & $\begin{array}{ll}0 & 0\end{array}$ & 0 & 1 & 0 & 0 & 0 & 0 & 0 & 0 & 0 & 0 & 0 & 0 & 0 & 0 & 0 & 0 & 0 \\
\hline$y_{3}$ & 0 & 0 & 0 & 0 & 0 & 0 & 0 & 0 & 0 & 0 & 0 & 0 & 0 & 0 & 0 & 0 & 0 & 0 & 0 & 0 & 0 \\
\hline$y_{4}$ & 0 & 0 & 0 & 0 & 0 & 0 & 0 & 0 & 0 & 0 & 0 & 0 & 0 & 0 & 0 & 0 & 0 & 0 & 0 & 0 & 0 \\
\hline$y_{5}$ & 0 & 0 & 0 & 0 & 0 & 0 & 0 & 0 & 0 & 0 & 0 & 0 & 0 & 0 & 0 & 0 & 0 & 0 & 0 & 0 & 0 \\
\hline$y_{6}$ & 0 & 0 & 0 & $\begin{array}{ll}0 & 0\end{array}$ & 0 & 0 & 0 & 0 & 0 & 0 & 0 & 0 & 0 & 0 & 0 & 0 & 0 & 0 & 0 & 0 & 0 \\
\hline$y_{7}$ & 0 & 0 & 0 & 0 & 0 & 0 & 1 & 1 & 0 & 1 & 0 & 1 & 1 & 0 & 0 & 0 & 0 & 0 & 0 & 0 & 0 \\
\hline$y_{8}$ & 0 & 0 & 0 & 0 & 0 & 0 & 0 & 1 & 0 & 1 & 0 & 0 & 1 & 0 & 1 & 1 & 1 & 1 & 0 & 1 & 0 \\
\hline$y_{9}$ & 0 & 1 & 0 & 0 & 0 & 0 & 0 & 1 & 1 & 1 & 1 & 1 & 1 & 0 & 1 & 1 & 1 & 1 & 0 & 1 & 0 \\
\hline$y_{10}$ & 0 & 0 & 1 & 0 & 0 & 0 & 0 & 0 & 1 & 1 & 1 & 1 & 1 & 0 & 1 & 1 & 1 & 1 & 0 & 1 & 0 \\
\hline$y_{11}$ & 0 & 0 & 0 & 1 & 0 & 0 & 0 & 1 & 0 & 1 & 0 & 1 & 1 & 0 & 1 & 1 & 1 & 1 & 0 & 1 & 0 \\
\hline$y_{12}$ & 1 & 0 & 0 & $\begin{array}{ll}0 & 0\end{array}$ & 0 & 0 & 0 & 1 & 0 & 1 & 0 & 1 & 1 & 0 & 1 & 1 & 1 & 1 & 0 & 1 & 0 \\
\hline$\left[\operatorname{Re}\left(\lambda_{i}\right)\right]$ & & & & .6598 & & & & & & & 4160 & & & & & & & .0000 & & & \\
\hline
\end{tabular}

were implemented using MPC technology. State-space models were developed according to the plant-model mismatch indicated by $\mathbf{z}^{\mathrm{n}}$ in Table 5 for the internal prediction in the MPC implementations. Additional details of the procedure being used are summarized in the Supporting Information file. The PIDs were tuned based on the IMC criterion and the parameters of the MPCs were selected following a trial and error procedure. It is worth to recall that there is no general recipe to tune the MPC parameters and, moreover, when the process size increases this becomes a very complex task. Figure 2 shows the main process variables, i.e., the reactor pressure and the $\mathrm{G} / \mathrm{H}$ product quality ratio, when the most important disturbance $\left(d_{1}\right)$ occurs as a step change at $t=0 \mathrm{~h}$. The dynamic responses of the three control structures are shown superimposed. In Fig. 2(a), considering the reactor pressure, it can be observed that the sparse MPC-based control structure presents the lowest peak value, which is far from the shutdown upper limit of $3000 \mathrm{kPa}$. In turn, the decentralized and full multivariable control structures have similar overshoots in this case, but still do not present any problem with the upper limit. This behavior is in accordance with the SSD+NLE combined index values reported in Table 3, where the lowest index is associated to the sparse control policy. Figure. 2(b) shows the G/H production ratio for the same disturbance $d_{1}$, where the sparse and decentralized control structure have the best performance 


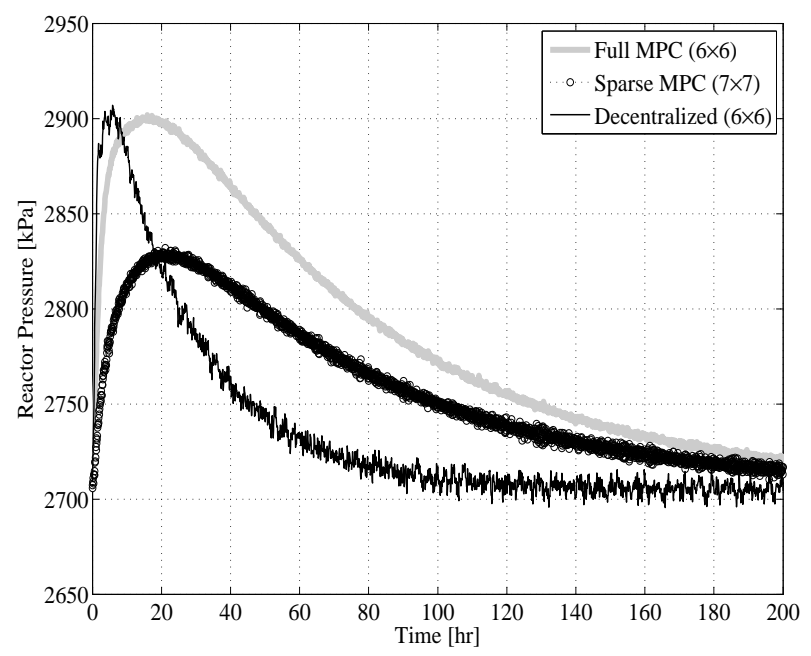

(a) Reactor Pressure $\left(y_{9}\right)$

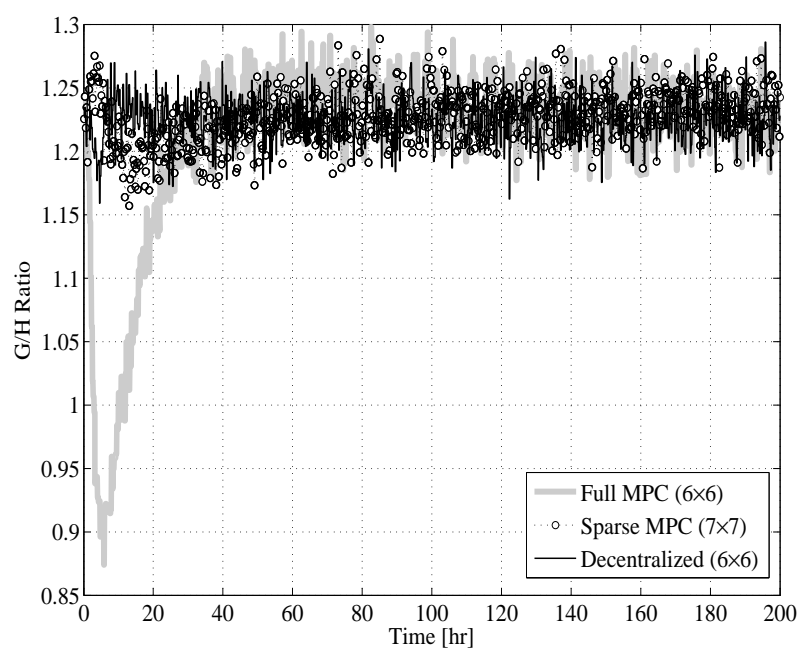

(b) G/H Ratio $\left(y_{12}\right)$

Figure 2: TEP - Dynamic responses for A/C composition disturbance $\left(d_{1}\right)$

without overshoot. This is a good example which shows that choosing a full MPC controller structure is not always the best choice (common practice). It is important to note that the MPC implementations have a very slow response compared to the decentralized PID-based control loops. This is basically due to the tuning parameters selected for the MPCs and their own interaction. While it is beyond the scope of this article, it is likely that a generalized and systematic tuning procedure, for example based on optimization, could find a better parameter set for these MPC.

\subsection{The waste water treatment plant}

The second case study, also taken from the literature, is a multivariable nonlinear benchmark developed within the framework of the European Co-Operation in the field of Scientific and Technical research (COST) Actions 682/624 and in collaboration with the IWA Task Group on Respirometry ${ }^{29}$. The overall layout of the waste water treatment plant (WWTP) is shown in Fig. 3, where five reactors (R1/R2 mixed-unaerated and R3/R4/R5 aerated) are presented in series with a 10-layer secondary settling tank (S). Two internal recycles exist in the process: the nitrate internal recycle $\left(Q_{i r}\right)$ from the fifth tank $(\mathrm{R} 5)$ to the first tank 


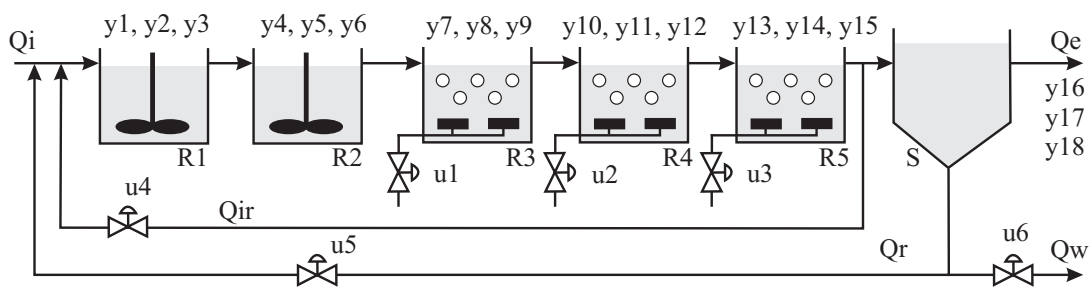

Figure 3: Waste water treatment plant (WWTP)

(R1), and recycle $Q_{r}$ from the underflow of the secondary settler (S) to the front end of the plant (no biological reaction occurs in the settler). The wastage $Q_{w}$ is pumped continuously from the underflow of the secondary settler. The main objective of the WWTP is to process the influent $Q_{i}$, with varying composition and flow, to give an effluent flow $Q_{e}$ with some specific quality index.

The main scenario used to apply the formulation proposed in Eq. 12 is summarized in Table 6 . In this case, $n=6$ available manipulated variables, $m=18$ potential controlled variables, and $p=6$ typical influent disturbances are considered. Besides, each reactor has 13 state variables. The original control requisites ${ }^{29}$ propose a basic $(2 \times 2)$ decentralized control policy with the following input-output pairing: $y_{5}-u_{4}$ and $y_{13}-u_{3}$. Hence, in this work the ad-hoc selection of $y_{5}$ and $y_{13}$ as CVs is maintained, being this fact indicated with an asterisk in Table 6 .

The MIQP algorithm proposed in eq. (12) has been applied to the WWTP considering the alternative decentralized, sparse, and full controller designs. Besides, according to Section 4.3, the original control requisites are fixed as $\mathbf{z}^{\mathrm{O}}(i)=1, \forall i=5,13$, and the selected range of feasible input-output pairing values is $\left[\delta_{1}, \delta_{2}\right]=[0.3,30]$. In all cases the big-M value is fixed to $M=30$. The results are summarized in Tables 7 to 9 . In Table 7 the model size, optimal objective function (i.e., the SSD+NLE index value), and required computational time for each controller structure are reported. This table shows that the most complex controller design scenario, the sparse case, only takes 2067.9 s to be solved. Taking into account that any entry of $\mathbf{z}^{\mathrm{n}}$ can potentially be selected, this is a very low optimization time for a large combinatorial problem. Besides, similar to the TEP case, here the sparse MCS design 
Table 6: WWTP variables

\begin{tabular}{|c|c|c|}
\hline & Inputs & Variable \\
\hline$u_{1}$ & Aeration reactor $3[-]$ & $K_{L} a 3$ \\
\hline$u_{2}$ & Aeration reactor $4[-]$ & $K_{L} a 4$ \\
\hline$u_{3}$ & Aeration reactor $5[-]$ & $K_{L} a 5$ \\
\hline$u_{4}$ & Internal recycle flow rate $\left[\mathrm{m}^{3} /\right.$ day $]$ & $Q_{i r}$ \\
\hline$u_{5}$ & Recycle flow rate $\left[\mathrm{m}^{3} /\right.$ day $]$ & $Q_{r}$ \\
\hline \multirow[t]{2}{*}{$u_{6}$} & Wastage flow rate $\left[\mathrm{m}^{3} /\right.$ day $]$ & $Q_{w}$ \\
\hline & Outputs & \\
\hline$y_{1}$ & Oxygen R1 [g COD m$\left.{ }^{3}\right]$ & $S_{O 1}$ \\
\hline$y_{2}$ & Nitrate and nitrite nitrogen $\mathrm{R} 1\left[\mathrm{~g} \mathrm{~N} \mathrm{~m}^{3}\right]$ & $S_{N O 1}$ \\
\hline$y_{3}$ & $\mathrm{NH}_{4}^{+}$and $\mathrm{NH}_{3}$ nitrogen $\mathrm{R} 1\left[\mathrm{~g} \mathrm{~N} \mathrm{~m}^{3}\right]$ & $S_{N H 1}$ \\
\hline$y_{4}$ & Oxygen R2 [g COD m³ $]$ & $S_{O 2}$ \\
\hline$y_{5}(*)$ & Nitrate and nitrite nitrogen $\mathrm{R} 2\left[\mathrm{~g} \mathrm{~N} \mathrm{~m}^{3}\right]$ & $S_{N O 2}$ \\
\hline$y_{6}$ & $\mathrm{NH}_{4}^{+}$and $\mathrm{NH}_{3}$ nitrogen $\mathrm{R} 2\left[\mathrm{~g} \mathrm{~N} \mathrm{~m}^{3}\right]$ & $S_{N H 2}$ \\
\hline$y_{7}$ & Oxygen R3 [g COD m³] & $S_{O 3}$ \\
\hline$y_{8}$ & Nitrate and nitrite nitrogen $\mathrm{R} 3\left[\mathrm{~g} \mathrm{~N} \mathrm{~m}^{3}\right]$ & $S_{N O 3}$ \\
\hline$y_{9}$ & $\mathrm{NH}_{4}^{+}$and $\mathrm{NH}_{3}$ nitrogen $\mathrm{R} 3\left[\mathrm{~g} \mathrm{~N} \mathrm{~m}^{3}\right]$ & $S_{N H 3}$ \\
\hline$y_{10}$ & Oxygen R4 [g COD m³] & $S_{O 4}$ \\
\hline$y_{11}$ & Nitrate and nitrite nitrogen $\mathrm{R} 4\left[\mathrm{~g} \mathrm{~N} \mathrm{~m}{ }^{3}\right]$ & $S_{N O 4}$ \\
\hline$y_{12}$ & $\mathrm{NH}_{4}^{+}$and $\mathrm{NH}_{3}$ nitrogen $\mathrm{R} 4\left[\mathrm{~g} \mathrm{~N} \mathrm{~m}^{3}\right]$ & $S_{N H 4}$ \\
\hline$y_{13}(*)$ & Oxygen R5 [g COD m$\left.{ }^{3}\right]$ & $S_{O 5}$ \\
\hline$y_{14}$ & Nitrate and nitrite nitrogen $\mathrm{R} 5\left[\mathrm{~g} \mathrm{~N} \mathrm{~m}^{3}\right]$ & $S_{N O 5}$ \\
\hline$y_{15}$ & $\mathrm{NH}_{4}^{+}$and $\mathrm{NH}_{3}$ nitrogen $\mathrm{R} 5\left[\mathrm{~g} \mathrm{~N} \mathrm{~m}^{3}\right]$ & $S_{N H 5}$ \\
\hline$y_{16}$ & Oxygen Qe $\left[\mathrm{g} \mathrm{COD} \mathrm{m}{ }^{3}\right]$ & $S_{O e}$ \\
\hline$y_{17}$ & Nitrate and nitrite nitrogen $\mathrm{Qe}\left[\mathrm{g} \mathrm{N} \mathrm{m}^{3}\right]$ & $S_{N O e}$ \\
\hline \multirow[t]{2}{*}{$y_{18}$} & $\mathrm{NH}_{4}^{+}$and $\mathrm{NH}_{3}$ nitrogen Qe $\left[\mathrm{g} \mathrm{N} \mathrm{m}^{3}\right]$ & $S_{N H e}$ \\
\hline & Disturbances & \\
\hline$d_{1}$ & Soluble inert organic matter $\left[\mathrm{g} \mathrm{COD} \mathrm{m}{ }^{3}\right]$ & $S_{I i}$ \\
\hline$d_{2}$ & Readily biodegradable substrate $\left[\mathrm{g} \mathrm{COD} \mathrm{m}^{3}\right.$ ] & $S_{S i}$ \\
\hline$d_{3}$ & Particulate inert organic matter $\left[\mathrm{g}\right.$ COD m$\left.{ }^{3}\right]$ & $X_{I i}$ \\
\hline$d_{4}$ & Slowly biodegradable substrate $\left[\mathrm{g} \mathrm{COD} \mathrm{m}^{3}\right.$ ] & $X_{S i}$ \\
\hline$d_{5}$ & Active heterotrophic biomass [g COD m${ }^{3}$ ] & $X_{B H i}$ \\
\hline$d_{6}$ & Influent flow rate $\left[\mathrm{m}^{3} /\right.$ day $]$ & $Q_{i}$ \\
\hline
\end{tabular}

represents the best controller structure with a cost function value of SSD+NLE $=13.46$.

Table 8 includes the selection of CVs and MVs for each controller structure, where $(3 \times 3)$, $(5 \times 5)$, and $(5 \times 5)$ multivariable control policies are selected by the optimization process for the decentralized, sparse, and full controllers, respectively. The best decentralized control policy defines $y_{5}, y_{10}$, and $y_{13}$ to be the CVs and $u_{3}, u_{4}$, and $u_{6}$ as the respective MVs, with the corresponding pairing shown in Table 9 (where $\mathbf{z}^{\mathrm{n}}=\mathbf{z}^{\text {nd }}$ ). Alternatively, the best sparse and full control structures involve the following selection of variables: $y_{5}, y_{7}, y_{10}$, 
Table 7: WWTP results - MIQP model size, cost function, and optimization time for each controller type

\begin{tabular}{l|r|r|r}
\cline { 2 - 4 } \multicolumn{1}{c|}{} & Decentralized & Sparse & Full \\
\hline Binary vars. & 130 & 238 & 238 \\
Continuous vars. & 3945 & 3945 & 3945 \\
Constraints & 18755 & 19157 & 19260 \\
Relaxed MIQP sol. & 4.971 & 4.971 & 4.971 \\
Cost function (SSD+NLE) & 18.41 & 13.46 & 15.13 \\
Optimization time [s] & 1891.81 & 2067.90 & 220.84 \\
\hline
\end{tabular}

$y_{13}$, and $y_{17}$ for the CVs and $u_{1}, u_{2}, u_{3}, u_{4}$, and $u_{6}$ for the MVs. In these cases, while the selection made by $\mathbf{z}^{\mathrm{O}}$ and $\mathbf{z}^{\mathrm{I}}$ is the same, the main difference lies in the plant-model mismatch indicated by $\mathbf{z}^{\mathrm{n}}$. Again, the entries highlighted with gray background in Table 9 represent the input-output pairing defined by $\mathbf{z}^{\text {nd }}$. In this table, the results of the off-line stability test, $\min \left[\operatorname{Re}\left(\lambda_{i}\right)\right]$, are also reported.

Similar to the previous case study, the optimal control structures for each controller type have been implemented and tested on the nonlinear dynamic WWTP benchmark using Matlab, and the same procedure outlined before has been applied for implementation and tuning. Figure 4 shows the main process variables when a dry weather disturbance is considered. This disturbance is a typical permanent diurnal variation in the influent flow and chemical oxygen demand (COD) load. In Figure 4, the dynamic responses of the three control structures are shown superimposed. Additionally, the basic control structure proposed in Copp ${ }^{29}$, which is labeled as "Decentralized $(2 \times 2)$ ", is included for comparison purposes. Figures. 4(a) and 4(b) display the original control requisites common to all the control structures, i.e., the nitrogen level in reactor $2\left(y_{5}\right)$ and the oxygen level in reactor 5 $\left(y_{13}\right)$. Although all the multivariable controllers maintain these CVs in their corresponding set points, the sparse and full MPC-based implementations present more variance. Figures 4(c) and 4(d) show two typical effluent quality indexes: the total nitrogen and total ammonia level, respectively. On the one hand, it can be observed in Fig. 4(c) that both the sparse and the decentralized $(3 \times 3)$ control structures present the best performance, minimizing the peak value and the violation time for the allowed limit of $18 \mathrm{gNm}^{-3}$. On the other hand, 
Table 8: WWTP results - Selection of CVs and MVs

\begin{tabular}{l|ccccccccccccccccccccccccccc}
\cline { 2 - 7 } & \multicolumn{11}{c|}{$\mathbf{z}^{\mathrm{O}}$} & \multicolumn{11}{c|}{$\mathbf{z}^{\mathrm{I}}$} \\
\cline { 2 - 3 } & $y_{1} y_{2}$ & $y_{3}$ & $y_{4}$ & $y_{5}$ & $y_{6}$ & $y_{7}$ & $y_{8}$ & $y_{9}$ & $y_{10}$ & $y_{11}$ & $y_{12}$ & $y_{13}$ & $y_{14}$ & $y_{15}$ & $y_{16}$ & $y_{17}$ & $y_{18}$ & $u_{1}$ & $u_{2}$ & $u_{3}$ & $u_{4}$ & $u_{5}$ & $u_{6}$ \\
\hline Decentralized & 0 & 0 & 0 & 0 & 1 & 0 & 0 & 0 & 0 & 1 & 0 & 0 & 1 & 0 & 0 & 0 & 0 & 0 & 0 & 0 & 1 & 1 & 0 & 1 \\
\hline Sparse & 0 & 0 & 0 & 0 & 1 & 0 & 1 & 0 & 0 & 1 & 0 & 0 & 1 & 0 & 0 & 0 & 1 & 0 & 1 & 1 & 1 & 1 & 0 & 1 \\
\hline Full & 0 & 0 & 0 & 0 & 1 & 0 & 1 & 0 & 0 & 1 & 0 & 0 & 1 & 0 & 0 & 0 & 1 & 0 & 1 & 1 & 1 & 1 & 0 & 1 \\
\hline
\end{tabular}

Table 9: WWTP results - Selected pairing $\left(\mathbf{z}^{\text {nd }}\right)$ and process-model mismatch $\left(\mathbf{z}^{\mathrm{n}}\right)$

\begin{tabular}{|c|c|c|c|c|c|c|c|c|c|c|c|c|c|c|c|c|c|c|}
\hline & \multicolumn{6}{|c|}{ Decentralized } & \multicolumn{6}{|c|}{ Sparse } & \multicolumn{6}{|c|}{ Full } \\
\hline & $u_{1}$ & $u_{2}$ & $u_{3}$ & & & & $u_{1}$ & $u_{2}$ & & & & $u_{6}$ & $u_{1}$ & $u_{2}$ & & & & \\
\hline$y_{1}$ & 0 & 0 & 0 & 0 & 0 & 0 & 0 & 0 & 0 & 0 & 0 & 0 & 0 & 0 & 0 & 0 & 0 & 0 \\
\hline$y_{2}$ & 0 & 0 & 0 & 0 & 0 & 0 & 0 & 0 & 0 & 0 & 0 & 0 & 0 & 0 & 0 & 0 & 0 & 0 \\
\hline$y_{3}$ & 0 & 0 & 0 & 0 & 0 & 0 & 0 & 0 & 0 & 0 & 0 & 0 & 0 & 0 & 0 & 0 & 0 & 0 \\
\hline$y_{4}$ & 0 & 0 & 0 & 0 & 0 & 0 & 0 & 0 & 0 & 0 & 0 & 0 & 0 & 0 & 0 & 0 & 0 & 0 \\
\hline$y_{5}$ & 0 & 0 & 0 & 1 & 0 & 0 & 0 & 1 & 0 & 1 & 0 & 1 & 1 & 1 & 1 & 1 & 0 & 1 \\
\hline$y_{6}$ & 0 & 0 & 0 & 0 & 0 & 0 & 0 & 0 & 0 & 0 & 0 & 0 & 0 & 0 & 0 & 0 & 0 & 0 \\
\hline$y_{7}$ & 0 & 0 & 0 & 0 & 0 & 0 & 1 & 1 & 0 & 1 & 0 & 1 & 1 & 1 & 1 & 1 & 0 & 1 \\
\hline$y_{8}$ & 0 & 0 & 0 & 0 & 0 & 0 & 0 & 0 & 0 & 0 & 0 & 0 & 0 & 0 & 0 & 0 & 0 & 0 \\
\hline$y_{9}$ & 0 & 0 & 0 & 0 & 0 & 0 & 0 & 0 & 0 & 0 & 0 & 0 & 0 & 0 & 0 & 0 & 0 & 0 \\
\hline$y_{10}$ & 0 & 0 & 0 & 0 & 0 & 1 & 1 & 1 & 0 & 1 & 0 & 1 & 1 & 1 & 1 & 1 & 0 & 1 \\
\hline$y_{11}$ & 0 & 0 & 0 & 0 & 0 & 0 & 0 & 0 & 0 & 0 & 0 & 0 & 0 & 0 & 0 & 0 & 0 & 0 \\
\hline$y_{12}$ & 0 & 0 & 0 & 0 & 0 & 0 & 0 & 0 & 0 & 0 & 0 & 0 & 0 & 0 & 0 & 0 & 0 & 0 \\
\hline$y_{13}$ & 0 & 0 & 1 & 0 & 0 & 0 & 1 & 0 & 1 & 1 & 0 & 0 & 1 & 1 & 1 & 1 & 0 & 1 \\
\hline$y_{14}$ & 0 & 0 & 0 & 0 & 0 & 0 & 0 & 0 & 0 & 0 & 0 & 0 & 0 & 0 & 0 & 0 & 0 & 0 \\
\hline$y_{15}$ & 0 & 0 & 0 & 0 & 0 & 0 & 0 & 0 & 0 & 0 & 0 & 0 & 0 & 0 & 0 & 0 & 0 & 0 \\
\hline$y_{16}$ & 0 & 0 & 0 & 0 & 0 & 0 & 0 & 0 & 0 & 0 & 0 & 0 & 0 & 0 & 0 & 0 & 0 & 0 \\
\hline$y_{17}$ & 0 & 0 & 0 & 0 & 0 & 0 & 0 & 1 & 1 & 1 & 0 & 0 & 1 & 1 & 1 & 1 & 0 & 1 \\
\hline$y_{18}$ & 0 & 0 & 0 & 0 & 0 & 0 & 0 & 0 & 0 & 0 & 0 & 0 & 0 & 0 & 0 & 0 & 0 & 0 \\
\hline $\min \left[\operatorname{Re}\left(\lambda_{i}\right)\right]$ & & & 0.55 & & & & & & 0.7 ? & 974 & & & & & & & & \\
\hline
\end{tabular}

Fig. 4(d) shows that the only control structure that does not violate the allowed ammonia limit of $4 \mathrm{gNm}^{-3}$ is the MPC-based sparse control. Furthermore, the decentralized $(2 \times 2)$ suggested by Copp ${ }^{29}$ presents the worst performance. The dynamic simulations allow to corroborate the conclusions obtained from the SSD+NLE optimization, i.e., the MCS with lower SSD+NLE index has the best dynamic performance. 


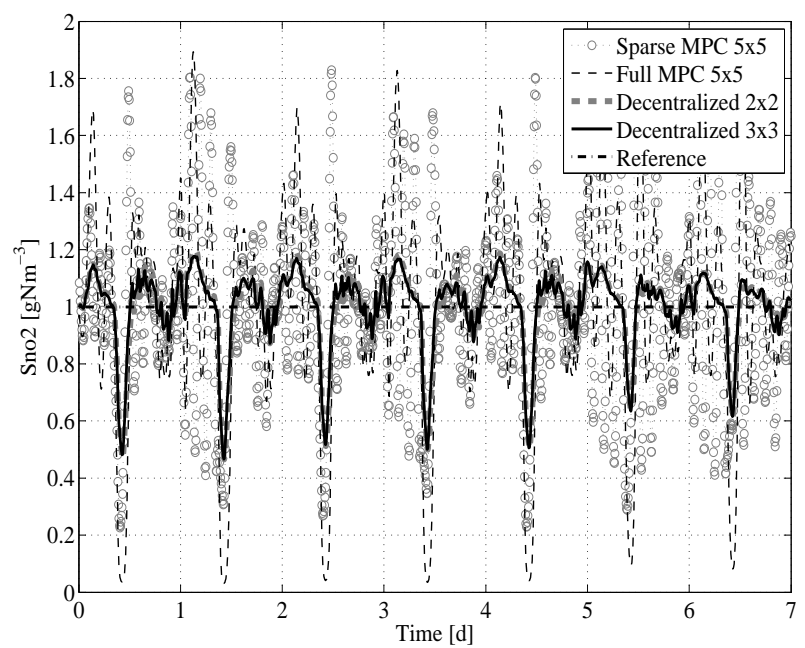

(a) Nitrogen reactor $2\left(y_{5}\right)$

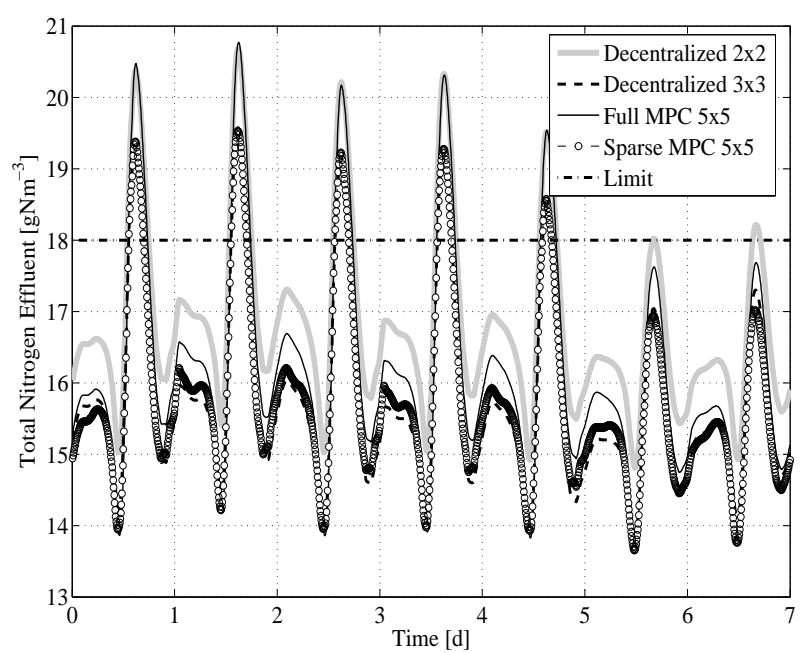

(c) Total nitrogen effluent

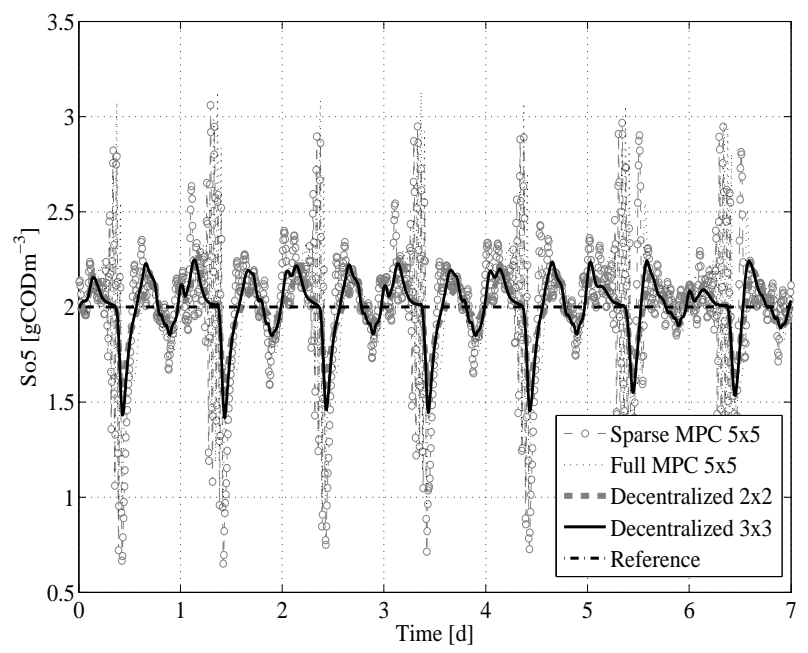

(b) Oxygen reactor $5\left(y_{13}\right)$

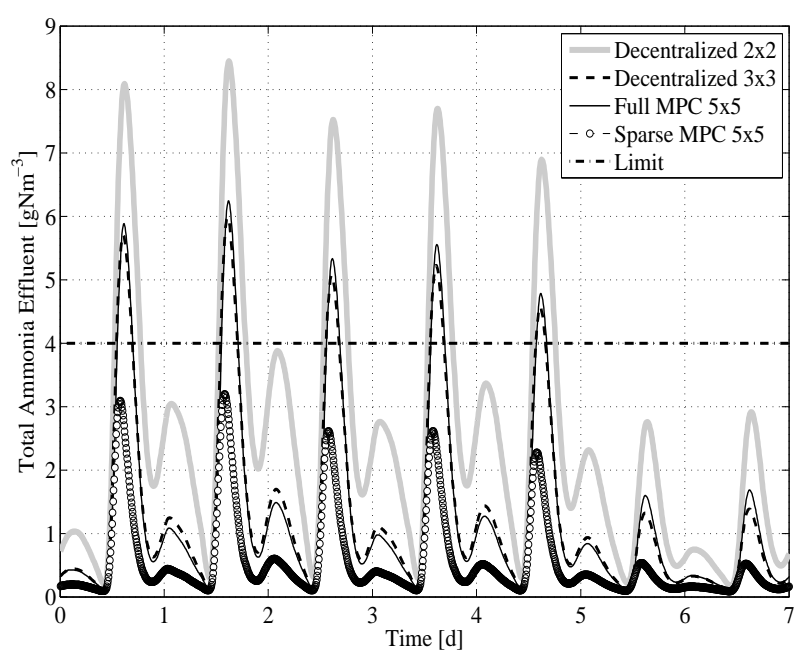

(d) Total ammonia effluent

Figure 4: WWTP - Dynamic responses for dry weather disturbance

\section{Conclusions}

In this work a mixed-integer quadratic programming (MIQP) model for multivariable control structure (MCS) design is proposed as a simplified reformulation of a previous bilevel mixed-integer nonlinear programming (BMINLP) superstructure ${ }^{12}$. The new representation allows to solve a very complex problem more suitably by using state-of-the-art solvers such as CPLEX in GAMS. Indeed, the new MIQP reformulation avoids to use non-convex constraints, which require complex global optimization algorithms (stochastic/deterministic) 
that present serious drawbacks related to optimality, computing time, and computational resources when the process size increases, becoming intractable for medium/large-scale plants. The proposed formulation is also convenient since there is a lack of rigorous global optimization algorithms for solving BMINLP models. To the best of our knowledge, the approach for MCS design presented here is the first equation-oriented representation that addresses simultaneously: the selection of $\mathrm{CVs}$ and $\mathrm{MVs}$, the input-output pairing definition, the controller design, the addition of original control requisites/heuristics, and a simplified stability/robustness test using steady-state information.

The proposed method has been successfully tested on two medium/large-scale benchmark case studies: the Tennessee Eastman Process (TEP) and the Waste Water Treatment Plant (WWTP), which represent real complex combinatorial problems. The results obtained by the MIQP reformulation using GAMS/CPLEX show that the optimization time can be reduced up to $94 \%$ compared with the GA-based MINLP approach. Moreover, the MIQP methodology guarantees that the global optimum is found, in contrast with the GA-based MINLP formulation which can be trapped in a local optimum. Three type of multivariable control structures based on three types of controller interaction were designed and successfully implemented. More precisely, optimal decentralized, sparse, and full controllers were designed for both case studies, showing that the classical heuristic decisions in MCS design (i.e., decentralized and full controllers) are not always the best choice. The test case results show that the optimal sparse MCS controllers have the best performance, in general, in accordance with the SSD+NLE index obtained by the MIQP formulation.

Furthermore, the proposed MIQP reformulation for MCS design sets the foundations for a proper future integration between process control and process synthesis and design. Indeed, because it includes only linear/convex constraints, the MIQP model is more effective and features lower computing times than other representations and, therefore, can be integrated with more complex formulations addressing process synthesis and/or design. 


\section{Acknowledgement}

The authors thank the financial support of CIFASIS-CONICET, ANPCYT, UNR and UTNFRRo from Argentina.

\section{Nomenclature}

\section{Acronyms}

BMINLP: Bilevel mixed-integer nonlinear programming

CVs: Controlled variables

DVs: Disturbances variables

GA: Genetic algorithms

IMC: Internal model control

MCS: Multivariable control structure

MINLP: Mixed-integer nonlinear programming

MIQP: Mixed-integer quadratic programming

MPC: Model predictive control

MSD: Minimum squared deviations

MVs: Manipulated variables

NLE: Net load evaluation

PS: Positive stable

PWC: Plantwide control

RGA: Relative gain array

SSD: Sum of squared deviations

TEP: Tennessee Eastman Process

TFM: Transfer function matrix

UVs: Uncontrolled variables

WWTP: Waste water treatment Plant

\section{Indexes}

$i=1, \ldots, m$ : Controlled variable

$j=1, \ldots, p:$ Perturbations

$l=1, \ldots, n:$ Manipulated variables

\section{Continuous variables}

$\mathbf{D}_{\mathrm{s}}$ : Disturbance TFM for CVs

$\mathbf{D}_{\mathrm{r}}$ : Disturbance TFM for UVs

$\mathbf{d}^{*}$ : Disturbance vector

$\mathbf{G}_{\mathrm{c}}$ : Controller TFM

$\mathbf{G}_{\mathrm{r}}$ : Process TFM for UVs

$\mathbf{G}_{\mathrm{s}}$ : Process TFM for CVs

$\tilde{\mathbf{G}}_{\mathrm{s}}$ : Particular selection of entries of $\mathbf{G}_{\mathrm{s}}$

NLE: Net load evaluation index

u: Input vector

$\mathbf{u}_{\mathrm{r}}$ : Not selected MVs vector

$\mathbf{u}_{\mathrm{s}}: \mathrm{MVs}$ vector

$\mathbf{u}_{\mathrm{s}}^{\mathrm{sp}}$ : MVs vector for set point changes of partitioning in eq. (3)

$\mathbf{u}_{\mathrm{s}}^{\mathrm{d}}$ : MVs vector for disturbance changes of partitioning in eq. (3)

$\mathbf{u}_{i}^{\mathrm{c}}$ : Complete input vector (including selected 
and not selected MVs) for the $i$-th set-point change

$\mathbf{u}_{j}^{\mathrm{d}}$ : Complete input vector for the $j$-th distur-

bance effects

$\mathbf{u}_{i}^{\text {nc }}$ : Complete input vector associated to $\mathbf{z}^{n}$ for

the $i$-th set-point change

$\mathbf{u}_{j}^{\text {nd }}$ : Complete input vector associated to $\mathbf{z}^{n}$ for

the $j$-th disturbance change

SSD: Sum of squared deviations index

y: Output vector

$\mathbf{y}_{\mathrm{r}}$ : UVs vector

$\mathbf{y}_{\mathrm{s}}: \mathrm{CVs}$ vector

$\mathbf{y}_{\mathrm{r}}^{\mathrm{d}}$ : UVs vector for disturbance effects of parti-

tioning in eq. (5)

$\mathbf{y}_{\mathrm{r}}^{\mathrm{sp}}$ : UVs vector for set point change of partitioning in eq. (5)

$\mathbf{y}_{\mathrm{s}}^{\mathrm{sp}}$ : CVs vector for set point change of partitioning in eq. (5)

$\mathbf{y}_{\mathrm{s}}^{\text {net}}$ : Net load effect vector of partitioning in

eq. (10)

$\mathbf{y}_{i}^{\mathrm{c}}$ : Complete output deviations (including CVs

and UVs) for the $i$-th set-point change

$\mathbf{y}_{j}^{\mathrm{d}}$ : Complete output deviations for the $j$-th dis-

turbance effects

$\mathbf{y}_{i}^{\text {nc: }}$ Complete net load effect vector associated

to $\mathbf{z}^{\mathrm{n}}$ for the $i$-th set-point change

$\mathbf{y}_{j}^{\text {nd }}$ : Complete net load effect vector associated

to $\mathbf{z}^{\mathrm{n}}$ for the $j$-th disturbance effects

$\mathbf{y}^{\mathrm{r}}$ : Complete RGA matrix (MIQP formulation)
$\Lambda_{\mathrm{p}}$ : Selected entries of the RGA matrix (original problem)

$\Lambda:$ RGA

$\Lambda_{n}:$ RGA-number

\section{Binary Variables}

$\mathbf{T}_{\mathrm{O}}, \mathbf{T}_{\mathrm{I}}$ : Transformation matrices (original problem)

$\mathbf{z}^{\mathrm{O}}$ : Vector for output variable selection

$\mathbf{z}^{\mathrm{I}}$ : Vector for input variable selection

$\mathbf{z}^{\mathrm{P}}$ : Matrix for decentralized control structure selection (original problem)

$\mathbf{z}^{\mathrm{S}}$ : Matrix for sparse control structure selection (original problem)

$\mathbf{z}^{\mathrm{n}}$ : Matrix for sparse control structure selection (MIQP formulation)

$\mathbf{z}^{\text {nd }}$ : Matrix for decentralized control structure selection (MIQP formulation)

\section{Parameters}

D: Disturbance TFM

F: Diagonal low-pass filter matrix

G: Process TFM

M: Big-M

$q$ : Number of unitary entries in $\mathbf{z}^{\mathrm{O}}$ and $\mathbf{z}^{\mathrm{I}}$ $\mathbf{v}_{i}, \mathbf{v}_{j}$ : Unit vectors of directions $i$ and $j$, respectively

$\delta_{1}, \delta_{2}$ : Lower and upper bounds for RGA entries selection (interaction range) 


\section{Appendix: Proposed MIQP model in scalar form}

Given the matrix $\mathbf{A}$ of size $(m \times n)$, the notation $\mathbf{A}=\left[a_{i, l}\right]$ is used to indicate that $a_{i, l}$ represents the $(i, l)$-th entry of the matrix A. The MIQP formulation in eq. (12) is presented in scalar form in Eq. (29), where the entries of the parameters and variables, either vectors or matrices, have the following characterization:

- parameters: $\mathbf{G}=\left[g_{k, l}\right]$ and $\mathbf{D}=\left[d_{k, j}\right]$,

- binary variables: $\mathbf{z}^{\mathrm{I}}=\left[z_{l}^{\mathrm{I}}\right], \mathbf{z}^{\mathrm{O}}=\left[z_{k}^{\mathrm{O}}\right], \mathbf{z}^{\mathrm{n}}=\left[z_{k, l}^{\mathrm{n}}\right]$, and $\mathbf{z}^{\mathrm{nd}}=\left[z_{k, l}^{\mathrm{nd}}\right]$,

- real (continuous) variables: $\mathbf{u}_{i}^{\mathrm{c}}=\left[u_{l, i}^{\mathrm{c}}\right], \mathbf{u}_{j}^{\mathrm{d}}=\left[u_{l, j}^{\mathrm{d}}\right], \mathbf{u}_{i}^{\mathrm{nc}}=\left[u_{i, k, l}^{\mathrm{nc}}\right], \mathbf{u}_{j}^{\mathrm{nd}}=\left[u_{j, k, l}^{\mathrm{nd}}\right], \mathbf{y}_{i}^{\mathrm{c}}=$ $\left[y_{i, k}^{\mathrm{c}}\right], \mathbf{y}_{j}^{\mathrm{d}}=\left[y_{j, k}^{\mathrm{d}}\right], \mathbf{y}_{i}^{\mathrm{nc}}=\left[y_{i, k}^{\mathrm{nc}}\right], \mathbf{y}_{j}^{\mathrm{nd}}=\left[y_{j, k}^{\mathrm{nd}}\right]$, and $\mathbf{y}^{\mathrm{r}}=\left[y_{i, l}^{\mathrm{r}}\right]$,

with $i=1, \ldots, m, k=1, \ldots, m, l=1, \ldots, n$, and $j=1, \ldots, p$. Besides, $\phi_{k, i}$ represents the $(k, i)$-th entry of the identity matrix $\mathbf{I}_{m}$. 


$$
\begin{aligned}
& \min _{\mathbf{u}, \mathbf{y}, \mathbf{z}} \sum_{i=1}^{m} \sum_{k=1}^{m}\left(y_{i, k}^{\mathrm{c}}\right)^{2}+\sum_{j=1}^{p} \sum_{k=1}^{m}\left(y_{j, k}^{\mathrm{d}}\right)^{2}+\sum_{i=1}^{m} \sum_{k=1}^{m}\left(y_{i, k}^{\mathrm{nc}}\right)^{2}+\sum_{j=1}^{p} \sum_{k=1}^{m}\left(y_{j, k}^{\mathrm{nd}}\right)^{2} \\
& \text { subject to } \quad-M\left(1-z_{k}^{\mathrm{O}}\right) \leq \sum_{l=1}^{n} g_{k, l} u_{l, i}^{\mathrm{c}}-\phi_{k, i} \leq M\left(1-z_{k}^{\mathrm{O}}\right), \quad \forall i, k \\
& -M\left(1-z_{k}^{\mathrm{O}}\right) \leq \sum_{l=1}^{n} g_{k, l} u_{l, j}^{\mathrm{d}}+d_{k, j} \leq M\left(1-z_{k}^{\mathrm{O}}\right), \quad \forall j, k \\
& -M z_{i}^{\mathrm{O}} \leq u_{l, i}^{\mathrm{c}} \leq M z_{i}^{\mathrm{O}}, \quad \forall i, l \\
& -M z_{l}^{\mathrm{I}} \leq u_{l, i}^{\mathrm{c}} \leq M z_{l}^{\mathrm{I}}, \quad \forall i, l \\
& -M z_{l}^{\mathrm{I}} \leq u_{l, j}^{\mathrm{d}} \leq M z_{l}^{\mathrm{I}}, \quad \forall j, l \\
& y_{i, k}^{\mathrm{c}}-\sum_{l=1}^{n} g_{k, l} u_{l, i}^{\mathrm{c}}+\phi_{k, i} z_{k}^{\mathrm{O}}=0, \quad \forall i, k \\
& y_{j, k}^{\mathrm{d}}-\sum_{l=1}^{n} g_{k, l} u_{l, j}^{\mathrm{d}}-d_{k, j}=0, \quad \forall j, k \\
& \sum_{i=1}^{m} z_{i}^{\mathrm{O}}=\sum_{l=1}^{n} z_{l}^{\mathrm{I}} \\
& \sum_{j=1}^{m} z_{j}^{\mathrm{O}} \leq q \\
& -M\left(1-z_{k, l}^{\mathrm{n}}\right) \leq u_{i, k, l}^{\mathrm{nc}}-u_{l, i}^{\mathrm{c}} \leq M\left(1-z_{k, l}^{\mathrm{n}}\right), \quad \forall i, k, l \\
& -M\left(1-z_{k, l}^{\mathrm{n}}\right) \leq u_{j, k, l}^{\mathrm{nd}}-u_{l, j}^{\mathrm{d}} \leq M\left(1-z_{k, l}^{\mathrm{n}}\right), \quad \forall j, k, l \\
& -M z_{k, l}^{\mathrm{n}} \leq u_{i, k, l}^{\mathrm{nc}} \leq M z_{k, l}^{\mathrm{n}}, \quad \forall i, k, l \\
& -M z_{k, l}^{\mathrm{n}} \leq u_{j, k, l}^{\mathrm{nd}} \leq M z_{k, l}^{\mathrm{n}}, \quad \forall j, k, l \\
& y_{i, k}^{\mathrm{nc}}+\sum_{l=1}^{n} g_{k, l} u_{i, k, l}^{\mathrm{nc}}-\phi_{k, i} z_{k}^{\mathrm{O}}=0, \quad \forall i, k \\
& y_{j, k}^{\mathrm{nd}}+\sum_{l=1}^{n} g_{k, l} u_{j, k, l}^{\mathrm{nd}}=0, \quad \forall j, k \\
& z_{k, l}^{\mathrm{n}} \leq z_{l}^{\mathrm{I}}, \quad z_{k, l}^{\mathrm{n}} \leq z_{k}^{\mathrm{O}}, \quad \forall k, l \\
& z_{l}^{\mathrm{I}} \leq \sum_{k=1}^{m} z_{k, l}^{\mathrm{n}}, \forall l, \quad z_{k}^{\mathrm{O}} \leq \sum_{l=1}^{n} z_{k, l}^{\mathrm{n}}, \forall k \\
& z_{l}^{\mathrm{I}}=\sum_{k=1}^{m} z_{k, l}^{\mathrm{nd}}, \forall l, \quad z_{k}^{\mathrm{O}}=\sum_{l=1}^{n} z_{k, l}^{\mathrm{nd}}, \forall k \\
& z_{k, l}^{\mathrm{nd}} \leq z_{k, l}^{\mathrm{n}}, \quad \forall k, l \\
& -M\left(1-z_{i, l}^{\mathrm{nd}}\right) \leq y_{i, l}^{\mathrm{r}}-g_{i, l} u_{l, i}^{\mathrm{c}} \leq M\left(1-z_{i, l}^{\mathrm{nd}}\right), \quad \forall i, l \\
& \delta_{1} z_{i, l}^{\mathrm{nd}} \leq y_{i, l}^{\mathrm{r}} \leq \delta_{2} z_{i, l}^{\mathrm{nd}}, \quad \forall i, l \\
& \sum_{i=1}^{m}\left(z_{i}^{\mathrm{O}}-y_{i, i}^{\mathrm{nc}}\right) \geq \delta
\end{aligned}
$$




\section{Supporting Information}

This material is available free of charge via the Internet at http://pubs.acs.org/.

The small-size generic example and the parameter settings for all the case studies are discussed in this supporting information file.

\section{References}

(1) Downs, J.; Skogestad, S. An industrial and academic perspective on plantwide control. Annu. Rev. Control 2011, 35, 99-110.

(2) Stephanopoulos, G.; Reklaitis, G. Process systems engineering: From Solvay to modern bio- and nanotechnology.: A history of development, successes and prospects for the future. Chem. Eng. Sci. 2011, 66, 4272-4306.

(3) Grosdidier, P.; Morari, M.; Holt, B. Closed-loop properties from steady-state gain information. Ind. Eng. Chem. Res. 1985, 24, 221-235.

(4) Skogestad, S.; Morari, M. Implications of large RGA elements on control performance. Ind. Eng. Chem. Res. 1987, 26, 2323-2330.

(5) Yuan, Z.; Chen, B.; Zhao, J. Effect of manipulated variables selection on the controllability of chemical processes. Ind. Eng. Chem. Res. 2011, 50, 7403-7413.

(6) Bristol, E. On a new measure of interaction for multivariable process control. IEEE Trans. Autom. Control 1966,

(7) Assali, W.; McAvoy, T. Optimal selection of dominant measurements and manipulated variables for production control. Ind. Eng. Chem. Res. 2010, 49, 7832-7842.

(8) Braccia, L.; Zumoffen, D. The input-output pairing problem: an optimization based approach. XVI Workshop on Information Processing and Control (RPIC). IEEE Conference Publications 2015, 60, 1-6. 
(9) Kadhim, A.; Castaño Arranz, M.; Birk, W. Automated control configuration selection considering system uncertainties. Ind. Eng. Chem. Res. 2017, 56, 3347-3359.

(10) Marchetti, A.; Zumoffen, D. Self-Optimizing Control Structures with Minimum Number of Process-Dependent Controlled Variables. Ind. Eng. Chem. Res. 2014, 53, 10177-10193.

(11) Zumoffen, D. Oversizing analysis in plant-wide control design for industrial processes. Comput. Chem. Eng. 2013, 59, 145-155.

(12) Zumoffen, D. Plant-wide control design based on steady-state combined indexes. ISA Trans. 2016, 60, 191-205.

(13) Sharifzadeh, M.; Thornhill, N. Optimal selection of control structure using a steadystate inverselly controlled process model. Comput. Chem. Eng. 2012, 39, 126-138.

(14) Rangaiah, G.; Kariwala, V. In Plantwide Control. Recent Developments and Applications; Rangaiah, G., Kariwala, V., Eds.; John Wiley and Sons Ltd: New York, 2012.

(15) Jha, A.; Okorafor, O. Optimal plantwide process control applied to the Tennessee Eastman problem. Ind. Eng. Chem. Res. 2014, 53, 738-751.

(16) Kookos, I.; Perkins, J. In The Integration of Process Design and Control; Seferlis, P., Georgiadis, M., Eds.; Computer Aided Chemical Engineering; Elsevier, 2004; Vol. 17; pp 216-238.

(17) Psaltis, A.; Kookos, I.; Kravaris, C. Plant-wide control structure selection methodology based on economics. Comput. Chem. Eng. 2013, 52, 240-248.

(18) Zumoffen, D.; Braccia, L.; Marchetti, A. Economic plant-wide control design with backoff estimations using internal model control. J. Process Control 2016, 40, 93-105. 
(19) Rafiei-Shishavan, M.; Mehta, S.; Ricardez-Sandoval, L. Simultaneous design and control under uncertainty: A back-offapproach using power series expansions. Comput. Chem. Eng. 2017, 99, 66-81.

(20) Gutierrez, G.; Ricardez-Sandoval, L.; Budman, H.; Prada, C. An MPC-based control structure selection approach for simultaneous process and control design. Comput. Chem. Eng. 2014, 70, 11-21.

(21) Ricardez-Sandoval, L.; Budman, H.; Douglas, P. Simultaneous design and control: A new approach and comparisons with existing methodologies. Ind. Eng. Chem. Res. 2010, 49, 2822-2833.

(22) Yue, D.; You, F. Stackelberg-game-based modeling and optimization for supply chain design and operations: A mixed integer bilevel programming framework. Comput. Chem. Eng. 2017, 102, 81-95.

(23) Saharidis, G.; Conejo, A.; Kozanidis, G. In Metaheuristics for bi-level optimization; Talbi, E., Ed.; Springer-Verlag: New York, 2013.

(24) Zumoffen, D.; Basualdo, M. Improvements on multiloop control design via net load evaluation. Comput. Chem. Eng. 2013, 50, 54-70.

(25) Garcia, C.; Morari, M. Internal model control. 2. Design procedure for multivariable systems. Ind. Eng. Chem. Process Des. Dev. 1985, 24, 472-484.

(26) Horn, R.; Johnson, C. Matrix analysis; Cambridge University Press: New York, 1990.

(27) Downs, J.; Vogel, E. A plant-wide industrial process control problem. Comput. Chem. Eng. 1992, 17, 245-255.

(28) McAvoy, T.; Ye, N. Base control for the Tennnessee Eastman challenge process. Comput. Chem. Eng. 1995, 19, 453-480. 
(29) Copp, J. The COST Simulation Benchmark: Description and Simulator Manual. IWA Task Group on Benchmarking of Control Strategies for WWTPs (http: // www. benchmarkwwtp. org/) 2000, p. 154. 


\section{TOC Graphic}

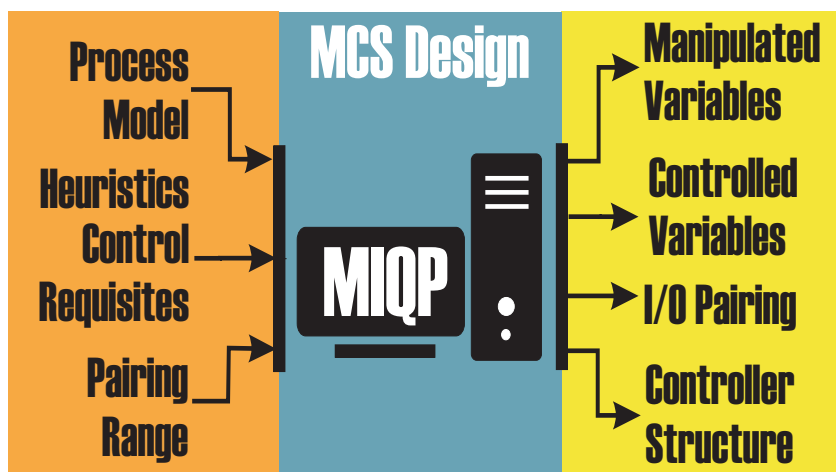

